\title{
The Baga-Gazriin Chuluu A-type granites of Central Mongolia compared with other igneous bodies nearby: a geochemical approach
}

\author{
Katarzyna MACHOWIAK and Wojciech STAWIKOWSKI
}

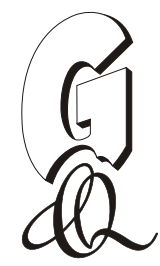

\begin{abstract}
Machowiak K. and Stawikowski W. (2012) - The Baga-Gazriin Chuluu A-type granites of Central Mongolia compared with other igneous bodies nearby: a geochemical approach. Geol. Quart., 56 (3): 457-474, doi: 10.7306/gq.1033

The subject of the study are the relationships between the Late Triassic A-type granites of the Baga-Gazriin Chuluu pluton in Central Mongolia and the magmatic bodies located either adjacent to or a short distance from this intrusion. We compare new geochemical data from the most representative samples of the Baga-Gazriin Chuluu pluton with published data from the Mesozoic granitoids of the Egiindavaa complex and Tsagduul massif in the Hotont area. The geochemical analyses of all these granitoids are also compared with the geochemical characteristics of trachyandesites of unknown age which neighbour the Baga-Gazriin pluton. The Baga-Gazriin granites, most of the Tsagduul granites and a few samples from Egiindavaa are A-granites with strong crustal affinity. By contrast, most of the Egiindavaa granitoids include considerable mantle-derived material and thus seemingly formed in different conditions, with a higher water content in the magma. The trachyandesites adjacent to the Baga-Gazriin Chuluu pluton have been referred to a Permian volcano-sedimentary complex, making them distinctly older than the granitoids. All the A-type granites discussed were emplaced in extensional conditions, which developed after cessation of the compressional regime resulting from the closure of the Mongol-Okhotsk Ocean. The shallow-lying source for their generation was connected with slab break-off or the presence of a hot spot. The granitoids, in the late stage of crystallisation, were enriched in complex ions that were REE carriers, as shown by a distinct "tetrad effect".
\end{abstract}

Katarzyna Machowiak, Institute of Civil Engineering, Poznań University of Technology, Piotrowo 5, 61-138 Poznań, Poland, e-mail: kamachow@amu.edu.pl; Wojciech Stawikowski, Institute of Geology, Adam Mickiewicz University, Maków Polnych 16, 61-606 Poznań, Poland, e-mail: wojst@amu.edu.pl (recevied: November 29, 2011; accepted: May 25, 2012; first published online: July 27, 2012).

Key words: Central Mongolia, Baga-Gazriin Chuluu pluton, A-type granites, geochemistry, Hotont area granitoids.

\section{INTRODUCTION}

Mongolia occupies a key position in the large-scale tectonic structure, termed the Central Asian Orogenic Belt (Windley et al., 2007), known also as the Altaids (Xiao et al., 2010). This continental-scale unit was formed as a result of the amalgamation of numerous tectonostratigraphic terranes of different geological histories (e.g., Badarch and Tomurtogoo, 2001). It has been regarded as a model example of an ancient accretionary orogen, i.e. the type of orogen connected with the growth of new continental crust (Cawood, 2009).

According to recent studies, (e.g., Mossakovsky et al., 1994; Badarch et al., 2002, Buchan et al., 2002, Yakubchuk, 2008), the present geological structure of Mongolia has been formed by complicated accretion and subduction processes taking place from the Neoproterozoic up to the Mesozoic. The traces of the terrane docking events are numerous tectonic sutures with frequently preserved fragments of oceanic litho- sphere in the form of ophiolitic sequences. The two main oceanic basins which closed during the formation of the Central Asian Orogenic Belt were the Paleo-Asian Ocean (Buslov et al., 2001) and the Mongol-Okhotsk Ocean (Kelty et al., 2008).

The remnant after the closure of the latter basin is the Mid-Mongolian Tectonic Line (MMTL), crossing through the centre of Mongolia and dividing its territory into northern and southern parts (Fig. 1). This lineament is marked by continuous set of individual faults which strike from the Mongolian Altai in the NW to the Döch Gol River valley in the NE (Tomurtogoo, 1997; Jahn et al., 2000). The timing, still debated, of the final convergence of the Mongol-Okhotsk Ocean is supposed to be Mid-Jurassic (Tomurtogoo et al., 2005), Late Jurassic (Zonenshain et al., 1990) or even around the Jurassic/Cretaceous boundary (Cogne et al., 2005). The closure of this oceanic basin, the minimum age of which is documented in Mongolia by the Carboniferous rocks of the Adaatsag ophiolite (Fig. 1) and Carboniferous detrital zircons from the Hangay-Hentey flysch basin (Kelty et al., 2008), was most 


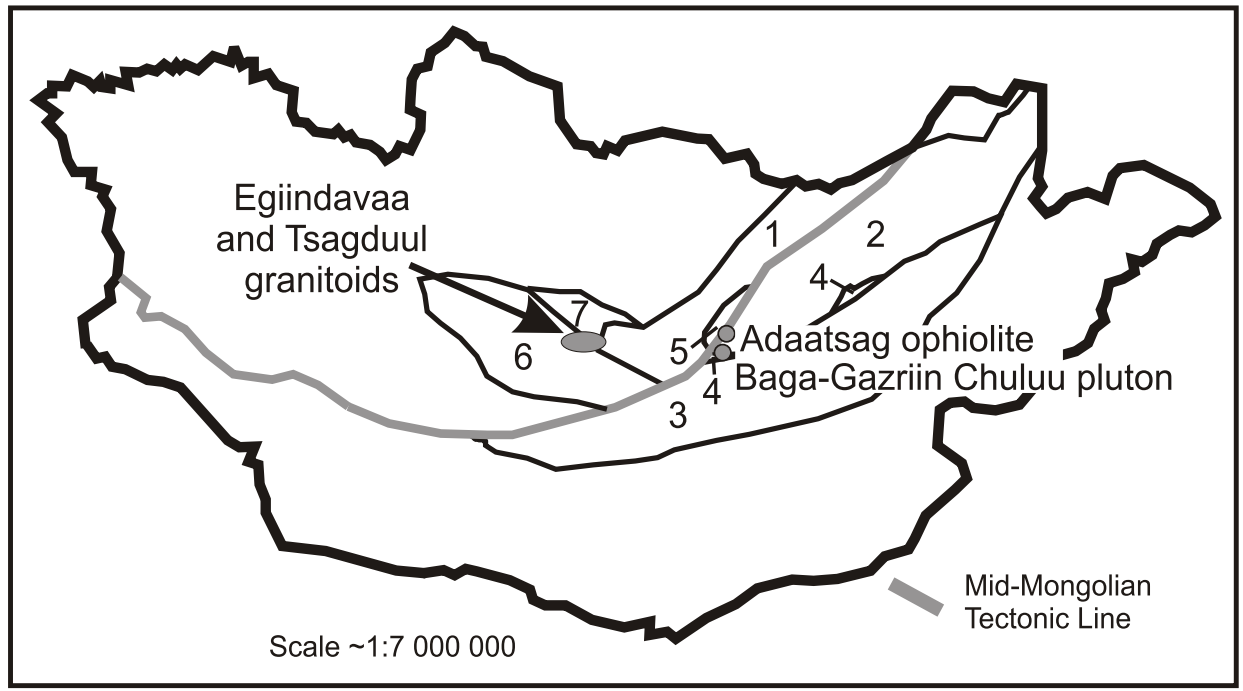

Fig. 1. Simplified map of NE Asia showing the position of the Mongol-Okhotsk suture (after Tomurtogoo et al., 2005, modified) and the location of the Baga-Gazriin Chuluu, Egiindavaa, Tsagduul granitoids and Adaatsag ophiolite

1 - Ulaanbataar terrane, 2 - Ereendavaa terrane, 3 - Idermeg terrane, 4 - Öndör Haan (Tsahir Uul) terrane, 5 - Adaatsag terrane, 6 - Tsetserleg terrane, 7 - Harhorin terrane

probably diachronous, continuing "in a scissors-like fashion" (Batulzii et al., 2003) from the west to the east.

For a complete understanding of accretionary processes in central and eastern Asia, there is a need for detailed information on the syn- and post-orogenic granitoids which are extraordinarily abundant in the Mongolian part of Central-Asian Orogenic Belt (Jahn et al., 2004; Kovalenko et al., 2004). This work, focusing on the geochemistry of the granitoid bodies situated close to the former suture zone, the Mid-Mongolian Tectonic Line, aims to contribute to knowledge of crustal growth in the Mongolian segment of the Central-Asian Orogenic Belt.

\section{GEOLOGICAL SETTING}

The Baga-Gazriin Chuluu (BGCh) granitic pluton is situated in Central Mongolia, ca. $220 \mathrm{~km}$ to the SSW from Ulaanbaatar and outcrops over an area of $c a$. $120 \mathrm{~km}^{2}$. It is located close to the merging point of three tectonic units (Fig. 1): the Adaatsag terrane, the Ereendavaa terrane and the Tsahir Uul subunit, which is a part of the Öndör Haan terrane (Tomurtogoo et al., 2005). It also belongs to the Middle Gobi volcano-plutonic belt distinguished by Badarch et al. (2002). The BGCh plutonic body lies close to the Mid-Mongolian Tectonic Line (MMTL), only $30 \mathrm{~km} \mathrm{SE}$ from the Adaatsag ophiolite and from the southern border of the Hangay-Hentey Basin, which represents the western part of the former Mongol-Okhotsk Ocean (Kelty et al., 2008).

So far, the age of the BGCh pluton has been established, based on correlation with other granitic bodies of the Hangay-Hentey Basin region, to be $\mathrm{T}_{3} / \mathrm{J}_{1}$ (e.g., Kovalenko et al., 1971). However, radiometric dating $\left({ }^{40} \mathrm{Ar} /{ }^{39} \mathrm{Ar}\right.$ technique, using biotite concentrates) showed the Late Triassic age of the BGCh granites (Machowiak and Stawikowski, pers. comm.).
In the Hotont area, $c a .120 \mathrm{~km}$ to the NW of the Baga-Gazriin Chuluu massif, still in the zone influenced by processes related to the formation of the MMTL lineament, Late Paleozoic Delgerhaan granitoids and Early Mesozoic Egiindavaa and Tsagduul granitoids are present (Oyungerel and Ishihara, 2005). The age of the two latter plutons based on the reconnaissance $\mathrm{Rb}$-Sr isotopic studies of Amar-Amgalan (2004, vide Oyungerel and Ishihara, 2005) has been established as Late Triassic to Early Jurassic. These Mesozoic granitoids display direct, usually intrusive contacts with the Paleozoic Delgerhaan granitoids.

The purpose of the paper is the comparison of new geochemical data obtained from the granites of the Baga-Gazriin Chuluu pluton with that from possibly genetically related Mesozoic intrusive rocks of the Hotont area (using published data of Oyungerel and Ishihara, 2005).

\section{PETROGRAPHY OF THE BAGA-GAZRIIN CHULUU PLUTON}

The Baga-Gazriin Chuluu (BGCh) pluton forms a sub-elliptical elevation with a maximum height of $1768 \mathrm{~m}$ a.s.l (Fig. 2), surrounded by flat topography of a volcano-sedimentary complex, that is considered to be Permian in age (Kovalenko et al., 1971).

The BGCh pluton is accompanied by numerous greisen veins. Its granites reveal also tungsten-tin mineralisation and enrichment in REE. No enclaves or relics after pyroxenes and amphibole have been observed.

The sedimentary part of the country rock sequence is built by sandstones, in part with intraclasts and locally muddy, whereas volcanogenic rocks of the complex are represented by ignimbrites, which can be found, for example, close to the SW 


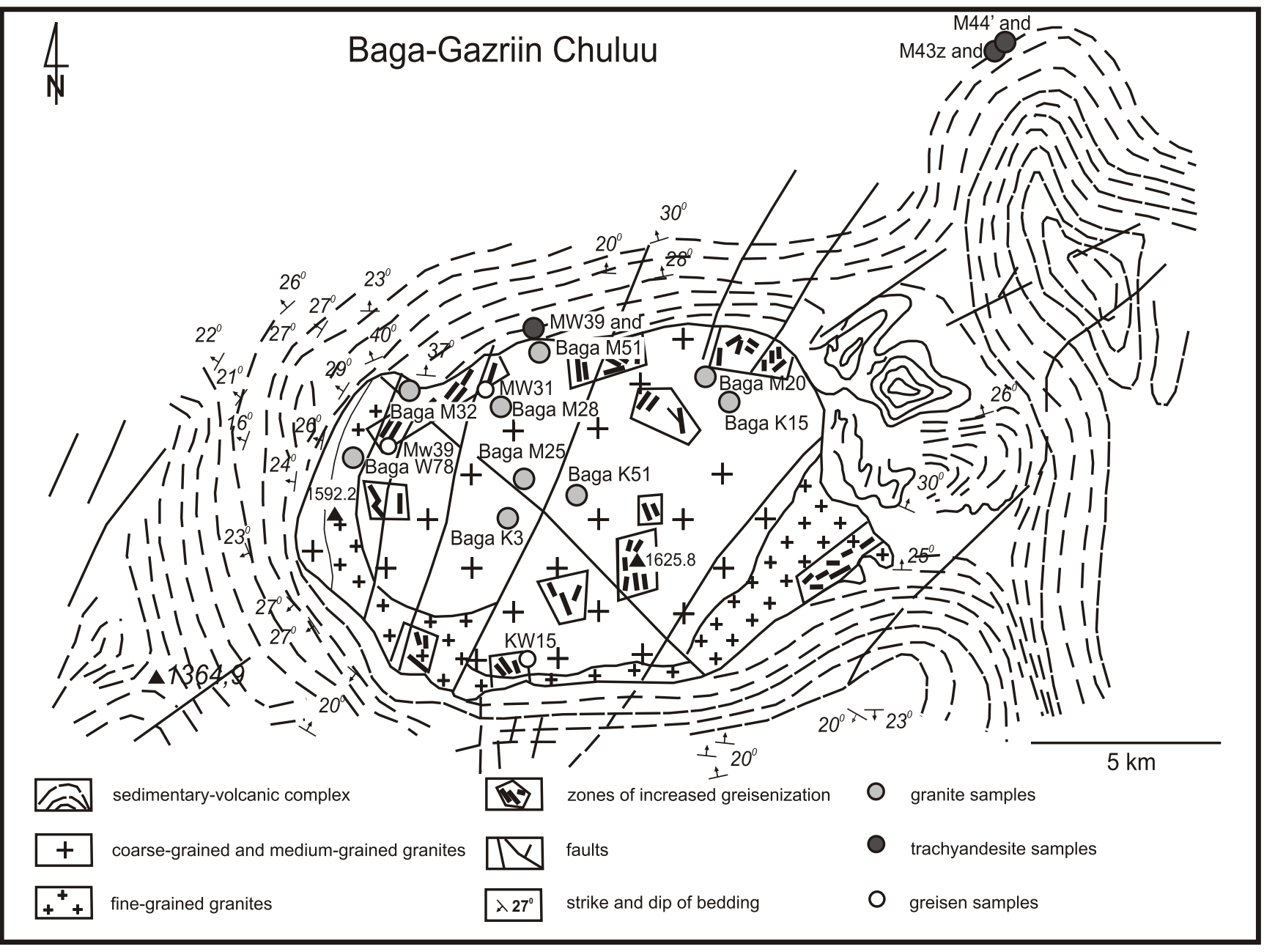

Fig. 2. Geological sketch of the Baga-Gazriin Chuluu pluton (after Kovalenko et al., 1971, modified)

margin of the pluton. Around the intrusion there occurs relatively a thin (up to several tens of metres) contact aureole with hornfelses (mainly with spotted texture).

In the direct vicinity of the magmatic massif, close to its north and north-west boundaries, as well as 10-20 km to NE, there can be found outcrops of dark trachyandesite (Fig. 2). Adjacent to the contact of the granitic body with the country rocks, the trachyandesites form scattered, small (several metres in diameter) outcrops. The lager ones, up to several tens of metres long, are situated ca. $10 \mathrm{~km}$ to the NE of the granites (Fig. 2).

\section{GRANITES}

We here divided the Baga-Gazriin Chuluu granites into three main textural varieties:

- coarse-grained, locally coarse-grained porphyritic granites - occurring mainly in the central part of the massif;

- medium-grained porphyritic granites - occurring across all the area studied;

- fine-grained equigranular granites (s.c. haplogranites) and fine-grained porphyritic granites (that occur in all the area studied, though predominantly in the marginal zone of the pluton).
The mineral composition of the BGCh granites is generally monotonous. They contain perthitic K-feldspar (usually ca. $90 \mathrm{~mol} . \%$ Or and up to $10 \mathrm{~mol} \% \mathrm{Ab}$ ), quartz, plagioclase (typically $90-100 \mathrm{~mol} . \% \mathrm{Ab}$ ), biotite and muscovite. In some of the samples, the lithium micas - zinnwaldite and lepidolite have been recognized. The granites studied include also high concentrations of topaz, fluorite and rare earth minerals, accompanied by the above-mentioned lithium micas. Their content increases with the intensity of greisenisation.

The K-feldspars in the porphyritic granites form two generations. The porphyrocrysts, mainly perthites, are usually automorphic or hypautomorphic, whereas the smaller grains are hypauto- or xenomorphic. Plagioclases are mostly zone-free and occur in subordinate amounts in part of the samples, they form only single grains. Micas include their dark and less frequently white types. Dark micas are represented by hypautomorphic biotite flakes (in the fresh granites) and usually xenomorphic grains of zinnwaldite (in the greisenised granites). Mostly hypautomorphic white micas have the composition of muscovite or lepidolite. They occur mainly in the greisenised samples, interstitialy or as the replacements after other minerals (mainly alkali feldspars and biotites). The modal content of the micas in the BGCh granites is strongly variable. In some of the samples biotite is accompanied by (generally 
secondary) muscovite or forms a paragenesis with lepidolite. Other samples contain only dark micas. Quartz is xenomorphic and usually, but not always, displays undulose extinction. Sporadically, myrmekite was observed.

Regarding accessory minerals, the most common are zircon and monazite. Other REE minerals have also been observed, however, their identification in thin sections was not possible. Depending on the level of greisenisation of the sampled granites, different contents (from accessory up to rock-forming levels) of topaz, fluorite and lithium micas aggregates can be identified. Zircon and monazite, usually fine-grained $(<50 \mathrm{mi}-$ crons), occur mainly in biotite. Hence, greater concentrations can be observed in the biotic granites.

Lithium micas and muscovite are usually devoid of these minerals or contain only their minute amounts. None of the granites show any alteration typical hydrothermal activity: thus, no chloritisation, serictisation or argillitisation was observed. The secondary alternations of the BGCh granites are mainly related to greisenisation processes.

\section{GREISENS}

The greisens of the Baga-Gazriin Chuluu pluton occur in the form of vein zones (Fig. 2) and, very sporadically, as patches resembling small lenses and enclaves. They can be divided into several types. In microscopic view, the particular types of greisen reveal considerable mineral diversity. The most frequent type are dark, mica-quartz greisens, with different proportions of quartz and micas. Their main components are xenomorphic, rarely hypautomorphic quartz and dark micas: biotite and zinnwaldite grouped into aggregates. Topaz and fluorite are also often observed in this greisen type, whereas feldspars are absent. The lighter quartz-mica greisens are dominated by quartz. Micas in this type are usually small or very small flakes concentrated into aggregates. The next type comprise quartz-fluorite greisens, with macroscopically visible violet and green fluorite mineralisation. These greisens contain only subordinate white micas. They are usually strongly fractured, which may be evidence of their genetic relation with the dislocation zones, what is suggested by the cataclasis that is visible also in thin sections.

The last main type of greisen microscopically resembles strongly greisenised granite. This type displays a texture inherited after granites and containing relics of magmatic minerals, intensively replaced by the greisenic paragenesis. The characteristic feature of this type is the preservation of significant amounts of perthite crystals. In some samples, completely developed pseudomorphs of lithium micas after megacrysts of alkaline feldspar can be found.

\section{TRACHYANDESITES}

The main constituents of the trachyandesites are plagioclase (18-26\% An), often albitised at the rims, K-feldspar (ca. 90 mol.\% Or, 10 mol.\% Ab), pyroxene (diopside), seemingly secondary amphibole (actinolite), titanomagnetite, post-biotitic chlorite, sphene and apatite.
Based on thin section studies, the trachyandesites can be divided into a fresh subtype, with well-preserved primary ferromagnesian minerals and an altered subtype, revealing post-magmatic changes resulting in a poorer mineral composition. The first subtype (Fig. 2; the outcrop $c a .10 \mathrm{~km}$ from the BGCh pluton) is characterized by a fine-grained matrix, the composition of which is identifiable under the microscope. It contains automorphic phenocrysts of polysynthetically twinned plagioclases surrounded by a matrix built of a second generation of plagioclases, pyroxenes, amphiboles, chlorite, sphene and oxides. The fresh trachyandesites register the onset of sericitisation, argillisation and chloritisation of its minerals. The second, altered subtype (Fig. 2, from the direct vicinity of the BGCh pluton) displays a porphyritic texture with a microcrystalline matrix. Its plagioclase phenocrysts are strongly sericitised. Clinopyroxenes and amphiboles are replaced by aggregates of chlorite and clay minerals.

\section{THE HOTONT AREA GRANITOIDS}

The Mesozoic granitoids of the Hotont area were described by Oyungerel and Ishihara (2005) as the Egiindavaa complex (EC) granitoids and the Tsagduul massif(TM) granites. They are intruded into the Delgerhaan granitic complex of Paleozoic age.

The Egiindavaa complex includes hornblende-biotite monzogranites, quartz monzodiorites with hornblende and biotite, fine- and medium-grained two-mica granites and haplogranites (Oyungerel and Ishihara, op. cit.). The Tsagduul massif granitoids are fine- to medium-grained mica granites with green, amazonitic K-feldspar and dark quartz. Both the EC and TM granitoids are considered as Mesozoic (Amar-Amgalan, 2004, vide Oyungerel and Ishihara, op. cit.) and are accompanied by greisens with tungsten-zinc and Ti-Ta-Nb mineralisation, which makes them similar to the BGCh granites.

\section{METHODS}

The samples from the BGCh pluton area, mainly granitic rocks of different types, including also greisens and trachyandesites, have been analysed for major and trace elements (including REE) in Activation Laboratories Ltd., Ontario, Canada, using international standards (more information about the procedures: http://www.actlabs.com/files/Euro_2011.pdf, page 16 and 17, code WRA + trace 4 Litho). The ten most representative analyses of the BGCh granites, three analyses of greisens and three analyses of trachyandesites from the BGCh area are presented in the paper (Table 1).

All the granitic rocks studied display elevated tungsten contents (355-544 ppm, av. $450 \mathrm{ppm}$ ). To estimate the level of possible artificial contamination of the samples, which could be the result of powdering the rocks with a tungsten carbide mill, an additional sterile sample (pure silica glass) was analysed. All the tungsten values given have been corrected taking into consideration the scale of contamination during sample prepara- 
Rock types and localities of the granitoids studied

\begin{tabular}{|c|c|c|c|c|}
\hline Research area & Sample no. & Longitude & Latitude & Rock name \\
\hline Baga-Gazriin Chuluu & Baga K51 & $106^{\circ} 05^{\prime} 13.3^{\prime \prime}$ & $46^{\circ} 10^{\prime} 38.0^{\prime \prime}$ & fine-grained granite \\
\hline Baga-Gazriin Chuluu & Baga W78 & $105^{\circ} 58^{\prime} 19.3^{\prime \prime}$ & $46^{\circ} 12^{\prime} 38.9^{\prime \prime}$ & fine-grained granite \\
\hline Baga-Gazriin Chuluu & Baga M32 & $105^{\circ} 59^{\prime} 53.9^{\prime \prime}$ & $46^{\circ} 13^{\prime} 50.0^{\prime \prime}$ & fine-grained granite \\
\hline Baga-Gazriin Chuluu & Baga M51 & $106^{\circ} 61^{\prime} 36.5^{\prime \prime}$ & $46^{\circ} 13^{\prime} 89.7^{\prime \prime}$ & medium-grained granite \\
\hline Baga-Gazriin Chuluu & Baga K15 & $106^{\circ} 05^{\prime} 26.6^{\prime \prime}$ & $46^{\circ} 13^{\prime} 38.4^{\prime \prime}$ & medium-grained granite \\
\hline Baga-Gazriin Chuluu & Baga M20 & $106^{\circ} 04^{\prime} 42.4^{\prime \prime}$ & $46^{\circ} 13^{\prime} 54.6^{\prime \prime}$ & medium-grained granite \\
\hline Baga-Gazriin Chuluu & Baga M28 & $106^{\circ} 01^{\prime} 10.1^{\prime \prime}$ & $46^{\circ} 13^{\prime} 25.2^{\prime \prime}$ & coarse-grained granite \\
\hline Baga-Gazriin Chuluu & Baga M25 & $106^{\circ} 00^{\prime} 48.1^{\prime \prime}$ & $46^{\circ} 12^{\prime} 55.9^{\prime \prime}$ & coarse-grained granite \\
\hline Baga-Gazriin Chuluu & Baga M31 & $105^{\circ} 59^{\prime} 21.0^{\prime \prime}$ & $46^{\circ} 13^{\prime} 59.9^{\prime \prime}$ & coarse-grained granite \\
\hline Baga-Gazriin Chuluu & Baga K3 & $106^{\circ} 02^{\prime} 20.8^{\prime \prime}$ & $46^{\circ} 12^{\prime} 14.5^{\prime \prime}$ & coarse-grained granite \\
\hline Baga-Gazriin Chuluu & KW5 & $106^{\circ} 03^{\prime} 30.7^{\prime \prime}$ & $46^{\circ} 12^{\prime} 53.0^{\prime \prime}$ & $\begin{array}{l}\text { gray greisen, fine-grained, with prevalent quartz, white mica } \\
\text { and accessory chlorite }\end{array}$ \\
\hline Baga-Gazriin Chuluu & KW15 & $106^{\circ} 00^{\prime} 26.3^{\prime \prime}$ & $46^{\circ} 10^{\prime} 24.9^{\prime \prime}$ & clear quartz-white mica greisen, fine-grained \\
\hline Baga-Gazriin Chuluu & MW31 & $106^{\circ} 00^{\prime} 45.0^{\prime \prime}$ & $46^{\circ} 13^{\prime} 25.4^{\prime \prime}$ & black biotite greisen, fine-grained \\
\hline Baga-Gazriin Chuluu & MW39 & $106^{\circ} 01^{\prime} 47.6^{\prime \prime}$ & $46^{\circ} 14^{\prime} 21.5^{\prime \prime}$ & trachyandesite \\
\hline Baga-Gazriin Chuluu & M43z & $106^{\circ} 08^{\prime} 94.4^{\prime \prime}$ & $46^{\circ} 15^{\prime} 07.5$ & trachyandesite \\
\hline Baga-Gazriin Chuluu & M44 & $106^{\circ} 08^{\prime} 94.4^{\prime \prime}$ & $46^{\circ} 15^{\prime} 07.5^{\prime \prime}$ & trachyandesite \\
\hline Egiindavaa complex & Egiin 14 & $102^{\circ} 08^{\prime} 39.0^{\prime \prime}$ & $47^{\circ} 12^{\prime} 43.0^{\prime \prime}$ & $\begin{array}{l}\text { porphyritic medium-grained hornblende-biotite granite } \\
\text { (authors: "quartz monzonite") }\end{array}$ \\
\hline Egiindavaa complex & Egiin 18 & $102^{\circ} 26^{\prime} 44.0^{\prime \prime}$ & $47^{\circ} 11^{\prime} 50.0^{\prime \prime}$ & fine-grained aplitic granite \\
\hline Tsagduul Massif & Tsagd 24 & $102^{\circ} 21^{\prime} 40.0^{\prime \prime}$ & $47^{\circ} 13^{\prime} 20.0^{\prime \prime}$ & fine-grained muscovite-biotite granite \\
\hline Tsagduul Massif & Tsagd 25 & $102^{\circ} 21^{\prime} 40.0^{\prime \prime}$ & $47^{\circ} 13^{\prime} 20.0^{\prime \prime}$ & very fine-grained aplitic granite \\
\hline
\end{tabular}

tion. For processing of the analytical results, the Geochemical Data Toolkit software (GCDkit; Janoušek et al., 2006) has been applied.

\section{GEOCHEMICAL CHARACTERISTICS OF THE BAGA-GAZRIIN CHULUU GRANITES IN RELATION TO THE ADJACENT TRACHYANDESITES AND THE HOTONT AREA GRANITES}

For the purpose of the presented comparative study, the ten most representative analyses of the BGCh granites, including the three textural varieties, have been selected (Table 1).

Results of the complete chemical analyses of three samples of BGCh pluton greisens and three samples of the trachyandesites from the close vicinity of the BGCh pluton are also included.

The second group of geochemical data analysed in the present paper are selected from the study of Oyungerel and Ishihara (2005; Table 3). Their data was exclusively from the granites assumed to be Mesozoic in age. Four chemical analyses from this paper have been used in this work; namely, the quartz monzodiorite (sample 14; the proper term according to the classification of De la Roche et al., 1980 is "quartz monzonite") and haplogranite (sample 18) from the Egiindavaa complex, the two-mica granite (sample Tsagd 24) and haplogranite (sample Tsagd 25) from the Tsagduul Massif (Table 2).

The synthetic diagrams (Figs. 3 and 4) include also data for major and selected trace elements of eight additional samples from the Hotont area (6 from Egiindavaa and 2 from
Tsagduul), for which the rare earth element contents were not published in the work of Oyungerel and Ishihara (op.cit.).

\section{THE CONCENTRATIONS OF MAJOR AND TRACE ELEMENTS}

The geochemistry of the Baga-Gazriin Chuluu (BGCh) granites is shown via a set of geochemical diagrams (Figs. 3-6 and 8-9), where the data for the BGCh granites are plotted together with the data for the granitoids of the Egiindavaa complex (EC) and the Tsagduul massif (TM). All the granitoids mentioned above were also compared with the trachyandesites, adjacent to the pluton studied in the BGCh area.

The EC granitoids are distinctly intrinsically variegated and contain different amounts of dark minerals, represented by biotite and amphiboles of the hornblende group (Oyungerel and Ishihara, 2005). The mineralogical diversity of the EC granitoids (based on the published data; op. cit) is reflected by their wide ranges of major element contents: $\mathrm{SiO}_{2}$ is between 66.1 and $77.35 \%, \mathrm{Al}_{2} \mathrm{O}_{3}$ 12.69-15.74, $\mathrm{Fe}_{2} \mathrm{O}_{3 \mathrm{t}} 0.63-3.53, \mathrm{MgO}$ 0.11-1.13, $\mathrm{CaO} 0.31-2.44, \mathrm{Na}_{2} \mathrm{O}$ 3.59-4.38, $\mathrm{K}_{2} \mathrm{O}$ 3.26-5.62, $\mathrm{TiO}_{2}$ 0.1-0.55, $\mathrm{P}_{2} \mathrm{O}_{5}$ 0.04-0.49.

In comparison to the Egiindavaa granitoids, the Baga-Gazriin Chuluu granites are much more homogenous. The BGCh granites are characterized by high levels of $\mathrm{SiO}_{2}$ (75.73-77.86\%) and $\mathrm{K}_{2} \mathrm{O}(4.45-5.18 \%)$. The contents of the remaining major elements range as follows: $\mathrm{Al}_{2} \mathrm{O}_{3} 11.0-13.01 \%$, $\mathrm{Fe}_{2} \mathrm{O}_{3 \mathrm{t}} 0.84-1.17 \%, \mathrm{MgO} 0.03-0.08 \%, \mathrm{CaO} 0.26-0.52 \%, \mathrm{Na}_{2} \mathrm{O}$ $3.01-3.86 \%$, $\mathrm{TiO}_{2} 0.048-0.115 \%, \mathrm{P}_{2} \mathrm{O}_{5}<0.01-0.17 \%$. (Table 2). 
Chemical composition of granitoids

\begin{tabular}{|c|c|c|c|c|c|c|c|c|c|c|}
\hline \multirow{2}{*}{ [wt.\%] } & \multicolumn{10}{|c|}{ Sample no. } \\
\hline & Baga K51 & Baga W78 & Baga M51 & Baga K15 & Baga M28 & Baga M25 & Baga M31 & Baga K3 & Baga M20 & Baga M32 \\
\hline $\mathrm{SiO}_{2}$ & 75.73 & 75.92 & 76.84 & 77.86 & 76.7 & 76.02 & 76.13 & 76.79 & 76.87 & 76.48 \\
\hline $\mathrm{TiO}_{2}$ & 0.058 & 0.069 & 0.07 & 0.068 & 0.096 & 0.063 & 0.048 & 0.115 & 0.083 & 0.08 \\
\hline $\mathrm{Al}_{2} \mathrm{O}_{3}$ & 12.58 & 12.49 & 12.15 & 11.0 & 11.64 & 12.17 & 13.01 & 12.25 & 11.63 & 11.83 \\
\hline $\mathrm{Fe}_{2} \mathrm{O}_{3 \mathrm{t}}$ & 1.17 & 1.1 & 0.95 & 0.96 & 1.03 & 0.89 & 0.84 & 1.21 & 1.09 & 1 \\
\hline $\mathrm{MnO}$ & 0.025 & 0.03 & 0.022 & 0.021 & 0.015 & 0.017 & 0.012 & 0.019 & 0.019 & 0.013 \\
\hline $\mathrm{MgO}$ & 0.03 & 0.03 & 0.04 & 0.04 & 0.05 & 0.04 & 0.03 & 0.08 & 0.06 & 0.03 \\
\hline $\mathrm{CaO}$ & 0.51 & 0.48 & 0.52 & 0.26 & 0.45 & 0.38 & 0.37 & 0.39 & 0.4 & 0.47 \\
\hline $\mathrm{Na}_{2} \mathrm{O}$ & 3.6 & 3.39 & 3.54 & 3.14 & 3.01 & 3.35 & 3.86 & 3.09 & 3.19 & 3.25 \\
\hline $\mathrm{K}_{2} \mathrm{O}$ & 4.61 & 4.61 & 4.84 & 4.45 & 4.96 & 4.7 & 4.54 & 5.18 & 4.75 & 4.79 \\
\hline $\mathrm{P}_{2} \mathrm{O}_{5}$ & b.d. & b.d. & 0.17 & 0.03 & b.d. & b.d. & 0.02 & 0.02 & b.d. & b.d. \\
\hline LOI & 0.89 & 0.74 & 0.74 & 0.79 & 0.79 & 0.95 & 0.96 & 0.73 & 0.58 & 0.71 \\
\hline $\mathrm{F}$ & b.d. & b.d. & b.d. & b.d. & b.d. & b.d. & b.d. & b.d. & b.d. & b.d. \\
\hline Total & 99.2 & 98.9 & 99.9 & 98.6 & 98.7 & 98.6 & 99.82 & 99.87 & 98.67 & 98.66 \\
\hline $\mathrm{A} / \mathrm{CNK}$ & 1.06 & 1.09 & 1.01 & 1.05 & 1.05 & 1.08 & 1.09 & 1.07 & 1.05 & 1.04 \\
\hline Mg-no. & 4.8 & 5.1 & 7.7 & 7.6 & 8.8 & 8.2 & 6.6 & 11.6 & 9.8 & 5.6 \\
\hline $\mathrm{T}_{\mathrm{Zr}}$ & 759 & 793 & 788 & 785 & 808 & 785 & 799 & 806 & 791 & 791 \\
\hline $\mathrm{AI}$ & 0.87 & 0.85 & 0.91 & 0.91 & 0.89 & 0.87 & 0.87 & 0.87 & 0.89 & 0.89 \\
\hline \multicolumn{11}{|l|}{ [ppm] } \\
\hline $\mathrm{Sc}$ & 3 & 5 & 4 & 3 & 4 & 3 & 3 & 5 & 3 & 3 \\
\hline $\mathrm{Be}$ & 7 & 8 & 11 & 7 & 8 & 5 & 6 & 8 & 9 & 6 \\
\hline $\mathrm{V}$ & b.d. & b.d. & b.d. & b.d. & b.d. & b.d. & b.d. & b.d. & b.d. & b.d. \\
\hline $\mathrm{Ba}$ & 10 & 21 & 71 & 25 & 52 & 58 & 56 & 156 & 67 & 37 \\
\hline $\mathrm{Sr}$ & 9 & 7 & 22 & 10 & 22 & 12 & 13 & 24 & 14 & 10 \\
\hline $\mathrm{Y}$ & 134 & 134 & 126 & 69 & 112 & 92 & 96 & 69 & 70 & 110 \\
\hline $\mathrm{Zr}$ & 100 & 143 & 147 & 129 & 175 & 130 & 152 & 166 & 144 & 147 \\
\hline $\mathrm{Cr}$ & b.d. & b.d. & b.d. & b.d. & b.d. & b.d. & b.d. & b.d. & b.d. & b.d \\
\hline $\mathrm{Co}$ & 99 & 77 & 83 & 85 & 83 & 67 & 86 & 70 & 80 & 92 \\
\hline $\mathrm{Ni}$ & b.d. & b.d. & b.d. & b.d. & b.d. & b.d. & b.d. & b.d. & b.d. & b.d. \\
\hline $\mathrm{Cu}$ & b.d. & b.d. & b.d. & b.d. & b.d. & b.d. & 60 & b.d. & b.d. & b.d. \\
\hline $\mathrm{Zn}$ & 40 & 60 & 50 & 30 & b.d. & b.d. & 40 & 50 & 30 & b.d. \\
\hline $\mathrm{Ga}$ & 24 & 26 & 25 & 20 & 26 & 26 & 29 & 22 & 26 & 27 \\
\hline $\mathrm{Rb}$ & 505 & 534 & 402 & 400 & 403 & 385 & 461 & 316 & 325 & 456 \\
\hline $\mathrm{Nb}$ & 54 & 73 & 40 & 34 & 50 & 40 & 51 & 29 & 35 & 48 \\
\hline Mo & b.d. & b.d. & b.d. & b.d. & b.d. & b.d. & b.d. & b.d. & b.d. & b.d. \\
\hline $\mathrm{Ag}$ & 0.6 & 0.8 & 0.5 & 0.7 & 0.7 & 0.6 & 2.3 & 0.9 & 0.6 & 0.8 \\
\hline $\mathrm{Sn}$ & 8 & 132 & 10 & 13 & 6 & 13 & 9 & 12 & 5 & 11 \\
\hline $\mathrm{Cs}$ & 13 & 21,7 & 21 & 16 & 11.4 & 13.1 & 14.3 & 15.8 & 8.8 & 11.4 \\
\hline $\mathrm{La}$ & 34.6 & 54.7 & 37 & 34.8 & 58.8 & 34.7 & 42.2 & 63.4 & 40.9 & 52.8 \\
\hline $\mathrm{Ce}$ & 97.8 & 119 & 88.7 & 74 & 124 & 78.5 & 98.1 & 145 & 89.7 & 114 \\
\hline $\operatorname{Pr}$ & 10.8 & 12.7 & 10.3 & 7.72 & 14.9 & 9.14 & 11.6 & 13.7 & 10.4 & 13.5 \\
\hline $\mathrm{Nd}$ & 39.3 & 43 & 38 & 25.7 & 49.9 & 31 & 39.4 & $\begin{array}{l}47.1 \\
\end{array}$ & 35.1 & 44.2 \\
\hline $\mathrm{Sm}$ & 11.9 & 10.9 & 9.7 & 6.1 & 12.1 & 8.1 & 10.4 & 9.5 & 8.4 & 10.8 \\
\hline $\mathrm{Eu}$ & 0.08 & 0.06 & 0.15 & 0.11 & 0.15 & 0.18 & 0.11 & 0.28 & 0.19 & 0.18 \\
\hline $\mathrm{Gd}$ & 12.9 & 11.8 & 10.2 & 6.4 & 11.4 & 8.1 & 9.4 & 8.9 & 7.8 & 10.3 \\
\hline $\mathrm{Tb}$ & 2.9 & 2.6 & 2.2 & 1.4 & 2.3 & 1.8 & 2 & 1.7 & 1.6 & 2.2 \\
\hline Dy & 19.7 & 18.4 & 15.3 & 9.4 & 16.4 & 13 & 14.5 & 11.1 & 10.9 & 15.7 \\
\hline Ho & 4.2 & 4.1 & 3.4 & 2.1 & 3.5 & 2.8 & 3.2 & 2.4 & 2.3 & 3.5 \\
\hline $\mathrm{Er}$ & 13.8 & 13.8 & 11.1 & 6.7 & 11.2 & 8.8 & 10.7 & 7.7 & 7.6 & 11.4 \\
\hline $\mathrm{Tm}$ & 2.38 & 2.51 & 1.95 & 1.18 & 1.99 & 1.48 & 1.89 & 1.32 & 1.23 & 1.98 \\
\hline $\mathrm{Yb}$ & 17 & 17.6 & 13.4 & 8.1 & 14.4 & 10.6 & 14 & 9.1 & 8.8 & 14.3 \\
\hline $\mathrm{Lu}$ & 2.73 & 2.8 & 2.16 & 1.27 & 2.34 & 1.77 & 2.28 & 1.45 & 1.44 & 2.28 \\
\hline
\end{tabular}


Tab. 2 cont.

\begin{tabular}{|c|c|c|c|c|c|c|c|c|c|c|}
\hline \multirow{2}{*}[\mathrm{ppm}]{} & \multicolumn{10}{|c|}{ Sample no. } \\
\hline & Baga K51 & Baga W78 & Baga M51 & Baga K15 & Baga M28 & Baga M25 & Baga M31 & Baga K3 & Baga M20 & Baga M32 \\
\hline $\mathrm{Hf}$ & 7.4 & 9.3 & 7.1 & 7 & 9.9 & 7.7 & 10.1 & 8.1 & 7.6 & 8.7 \\
\hline $\mathrm{Ta}$ & 8.2 & 9.4 & 5.2 & 4.2 & 6.3 & 4.9 & 6.2 & 4.6 & 5.3 & 6.5 \\
\hline $\mathrm{W}$ & 544 & 460 & 450 & 414 & 474 & 355 & 500 & 361 & 432 & 576 \\
\hline $\mathrm{Tl}$ & 2.8 & 2.9 & 2 & 1.9 & 2.5 & 2.3 & 2.6 & 1.5 & 1.6 & 2.7 \\
\hline $\mathrm{Pb}$ & 49 & 28 & 43 & 33 & 32 & 27 & 32 & 37 & 40 & 27 \\
\hline Th & 38.7 & 45.8 & 36.3 & 26.5 & 47.1 & 40.5 & 58.4 & 27.1 & 37.2 & 46.1 \\
\hline $\mathrm{U}$ & 3.4 & 9.6 & 5.2 & 6.1 & 14.4 & 4.8 & 7.1 & 11.6 & 4.4 & 7.3 \\
\hline$\sum$ REE & 236.2 & 314 & 243.6 & 185 & 323.4 & 210 & 260 & 322.7 & 226.4 & 297.1 \\
\hline LREE & 194.48 & 240.36 & 183.85 & 148,43 & 259.85 & 161.62 & 201.81 & 278.98 & 184.69 & 235.48 \\
\hline HREE & 41.67 & 73.61 & 59.71 & 36.55 & 63.53 & 48.35 & 57.95 & 43.67 & 41.67 & 61.66 \\
\hline $\begin{array}{l}\text { LREE } \\
\text { /HREE }\end{array}$ & 4.7 & 3.3 & 3.1 & 4.1 & 4.1 & 3.3 & 3.5 & 6.4 & 4.4 & 3.8 \\
\hline $\mathrm{TE}_{1,3}$ & 1.2 & 1.13 & 1.13 & 1.13 & 1.11 & 1.15 & 1.14 & 1.11 & 1.13 & 1.13 \\
\hline $\mathrm{Eu} / \mathrm{Eu}^{*}$ & 0.02 & 0.02 & 0.05 & 0.05 & 0.04 & 0.07 & 0.03 & 0.09 & 0.07 & 0.05 \\
\hline
\end{tabular}

b.d. - below detection limit

$\mathrm{Tab} 1 \mathrm{e} 3$

Chemical composition of granitoids, greisens and trachyandesites

\begin{tabular}{|c|c|c|c|c|c|c|c|c|c|c|}
\hline \multirow{2}{*}{ [wt.\%] } & \multicolumn{10}{|c|}{ Sample no. } \\
\hline & Egiin 14 & Egiin 18 & Tsagd 24 & Tsagd 25 & KW5 grs & KW15 grs & MW31 grs & MW39 and & M43z and & M44' and \\
\hline $\mathrm{SiO}_{2}$ & 66.1 & 75.19 & 74.6 & 77.15 & 75.35 & 89.66 & 55.77 & 54.02 & 54.85 & 55.19 \\
\hline $\mathrm{TiO}_{2}$ & 0.55 & 0.11 & 0.16 & 0.02 & 1.64 & 0.069 & 0.276 & 1.549 & 1.495 & 1.497 \\
\hline $\mathrm{Al}_{2} \mathrm{O}_{3}$ & 15.74 & 13.18 & 13.85 & 12.76 & 13.57 & 9.55 & 17.03 & 17.71 & 17.56 & 16.22 \\
\hline $\mathrm{Fe}_{2} \mathrm{O}_{3 \mathrm{t}}$ & 3.53 & 1.13 & 1.12 & 0.75 & 4.56 & 0.16 & 13.21 & 8.75 & 8.41 & 8.87 \\
\hline $\mathrm{MnO}$ & 0.07 & 0.02 & 0.05 & 0.02 & 0.072 & 0.003 & 0.564 & 0.13 & 0.118 & 0.139 \\
\hline $\mathrm{MgO}$ & 1.13 & 0.21 & 0.2 & 0.03 & 0.06 & 0.01 & 0.19 & 2.26 & 2.34 & 2.78 \\
\hline $\mathrm{CaO}$ & 2.39 & 0.65 & 0.84 & 0.16 & 0.98 & 0.05 & 0.61 & 5.76 & 5.5 & 2.76 \\
\hline $\mathrm{Na}_{2} \mathrm{O}$ & 4.2 & 3.59 & 3.97 & 4.18 & 0.03 & 0.03 & 0.05 & 3.21 & 3.53 & 4.31 \\
\hline $\mathrm{K}_{2} \mathrm{O}$ & 4.4 & 5.1 & 5.04 & 4.59 & 1.64 & 0.1 & 7.39 & 2.58 & 2.74 & 3.07 \\
\hline $\mathrm{P}_{2} \mathrm{O}_{5}$ & 0.49 & 0.05 & 0.07 & 0.05 & 0.04 & 0.03 & 0.07 & 0.64 & 0.64 & 0.68 \\
\hline LOI & 1.1 & 0.64 & 0.19 & 0.19 & 2.33 & 1.25 & 3.27 & 1.93 & 2.79 & 3.12 \\
\hline $\mathrm{F}$ & b.d. & b.d. & 0.12 & 0.39 & b.d. & b.d. & b.d. & b.d. & b.d. & b.d. \\
\hline Total & 99.8 & 99.9 & 100.1 & 99.9 & 98.74 & 100.9 & 98.43 & 98.6 & 99.9 & 98.6 \\
\hline $\mathrm{A} / \mathrm{CNK}$ & 0.98 & 1.05 & 1.02 & 1.05 & - & - & - & - & - & - \\
\hline Mg-no. & 38.8 & 26.9 & 26.1 & 33.8 & - & - & - & 33.8 & 35.5 & 38.3 \\
\hline $\mathrm{T}_{\mathrm{Zr}}$ & 836 & 804 & 753 & 811 & - & - & - & - & - & - \\
\hline AI & 0.74 & 0.87 & 0.87 & 0.93 & - & - & - & - & - & - \\
\hline \multicolumn{11}{|l|}{$[\mathrm{ppm}]$} \\
\hline $\mathrm{Sc}$ & b.d. & b.d. & b.d. & b.d. & 4 & 2 & 22 & 22 & 19 & 19 \\
\hline $\mathrm{Be}$ & b.d. & b.d. & b.d. & b.d. & 8 & b.d. & 10 & 8 & 3 & 2 \\
\hline $\mathrm{V}$ & 34 & 18 & 7 & b.d. & b.d. & b.d. & 14 & 209 & 207 & 189 \\
\hline $\mathrm{Ba}$ & 823 & 808 & 265 & b.d. & 39 & 5 & 102 & 770 & 896 & 1279 \\
\hline $\mathrm{Sr}$ & 384 & 284 & 99 & 2 & 12 & 3 & 31 & 596 & 799 & 359 \\
\hline $\mathrm{Y}$ & 28.6 & 17.2 & 21.8 & 116 & 99 & 24 & 58 & 48 & 39 & 42 \\
\hline $\mathrm{Zr}$ & 368 & 177 & 103 & 174 & 195 & 159 & 199 & 371 & 346 & 385 \\
\hline $\mathrm{Cr}$ & 45 & 22 & 39 & 12 & b.d. & b.d. & b.d. & b.d. & b.d. & b.d. \\
\hline $\mathrm{Co}$ & 5 & 3 & b.d. & b.d. & 100 & 122 & 24 & 30 & 27 & 27 \\
\hline $\mathrm{Ni}$ & 6.2 & 1.9 & 1.3 & 3.8 & b.d. & b.d. & b.d. & 40 & b.d. & b.d. \\
\hline $\mathrm{Cu}$ & 5.1 & b.d. & b.d. & b.d. & 400 & 180 & 20 & 90 & 130 & 100 \\
\hline $\mathrm{Zn}$ & 38 & b.d. & 38 & 197 & 340 & 80 & 280 & 240 & 100 & 100 \\
\hline $\mathrm{Ga}$ & 23 & 19 & 23 & 47 & 29 & 5 & 69 & 23 & 21 & 18 \\
\hline
\end{tabular}




\begin{tabular}{|c|c|c|c|c|c|c|c|c|c|c|}
\hline \multirow{2}{*}{ [ppm] } & \multicolumn{10}{|c|}{ Sample no. } \\
\hline & Egiin14 & Egiin 18 & Tsagd 24 & Tsagd 25 & KW5 grs & KW15 grs & MW31 grs & MW39 and & $\mathrm{M} 43 \mathrm{z}$ and & M44' and \\
\hline $\mathrm{Rb}$ & 210 & 137 & 294 & 1.12 & 506 & 14 & $>1000$ & 218 & 78 & 108 \\
\hline $\mathrm{Nb}$ & 15.9 & 10 & 21.5 & 65.3 & 37 & 45 & 90 & 15 & 14 & 13 \\
\hline Mo & 1.6 & 0.6 & 0 & 0 & 3 & b.d. & 5 & b.d. & b.d. & b.d. \\
\hline $\mathrm{Ag}$ & b.d. & b.d. & b.d. & b.d. & 0.8 & 5.7 & 1.8 & 2.4 & 1.6 & 1.8 \\
\hline $\mathrm{Sn}$ & 5 & 3 & 6 & 147 & $>1000$ & 93 & 92 & 37 & 3 & 2 \\
\hline $\mathrm{Cs}$ & 13.9 & 7.6 & 14.5 & 29.5 & 39.5 & 0.7 & 123 & 40 & 4.1 & 5 \\
\hline $\mathrm{La}$ & 69 & 38.7 & 34.9 & 16.6 & 59.2 & 14.8 & 170 & 34.6 & 41.6 & 39.1 \\
\hline $\mathrm{Ce}$ & 136 & 61.5 & 67 & 48.7 & 138 & 37.8 & 374 & 70.4 & 90.8 & 92.8 \\
\hline $\operatorname{Pr}$ & 14.9 & 7.52 & 7.01 & 5.98 & 14.2 & 4.03 & 43 & 10.1 & 11.2 & 11.3 \\
\hline $\mathrm{Nd}$ & 52.6 & 25.8 & 24.2 & 25.2 & 49.4 & 13.7 & 137 & 42.8 & 43.6 & 46.8 \\
\hline $\mathrm{Sm}$ & 8.74 & 4.16 & 4.24 & 7.05 & 11.7 & 3 & 23.5 & 9.9 & 9.2 & 9.9 \\
\hline $\mathrm{Eu}$ & 1.45 & 0.689 & 0.523 & 0.006 & 0.27 & 0.09 & 0.3 & 1.88 & 1.86 & 1.87 \\
\hline $\mathrm{Gd}$ & 6.33 & 3.29 & 3.2 & 7.38 & 11.1 & 2.4 & 12.7 & 8.8 & 7.9 & 8.8 \\
\hline $\mathrm{Tb}$ & 0.94 & 0.49 & 0.52 & 1.97 & 2.3 & 0.5 & 1.8 & 1.4 & 1.3 & 1.4 \\
\hline Dy & 4.76 & 2.57 & 2.92 & 15.7 & 14.7 & 3.5 & 10.2 & 8.5 & 7.1 & 7.8 \\
\hline Ho & 0.88 & 0.53 & 0.6 & 3.84 & 3.1 & 0.8 & 2.2 & 1.7 & 1.4 & 1.5 \\
\hline $\mathrm{Er}$ & 2.68 & 1.64 & 2 & 14.4 & 9.9 & 2.7 & 7.3 & 5.2 & 3.9 & 4.3 \\
\hline $\mathrm{Tm}$ & 0.415 & 0.274 & 0.36 & 2.99 & 1.69 & 0.49 & 1.18 & 0.76 & 0.57 & 0.64 \\
\hline $\mathrm{Yb}$ & 2.69 & 1.81 & 2.59 & 22.6 & 11.9 & 3.5 & 7.9 & 4.9 & 3.8 & 4.2 \\
\hline $\mathrm{Lu}$ & 0.395 & 0.28 & 0.435 & 3.35 & 1.93 & 0.56 & 1.23 & 0.78 & 0.63 & 0.68 \\
\hline Hf & 9.9 & 5.2 & 4.2 & 21.6 & 8.1 & 7.1 & 9.6 & 10.1 & 10.5 & 10.4 \\
\hline $\mathrm{Ta}$ & 1.6 & 1.2 & 3.9 & 17.6 & 3.9 & 4.8 & 7.9 & 0.8 & 0.7 & 0.7 \\
\hline $\mathrm{W}$ & 1.5 & 0.9 & 2.4 & 1.5 & 609 & 945 & b.d. & b.d. & b.d. & b.d. \\
\hline $\mathrm{Tl}$ & 0.9 & 0.7 & 1.6 & 5.9 & 3.3 & 0.2 & 4.6 & 2.4 & 0.2 & 0.3 \\
\hline $\mathrm{Pb}$ & 26 & 31 & 41 & 104 & b.d. & 9 & 65 & 7 & 19 & 20 \\
\hline $\mathrm{Bi}$ & 0.7 & 1.1 & 0.4 & 3.8 & b.d. & 125 & 123 & 0.7 & b.d. & b.d. \\
\hline Th & 52.6 & 20.7 & 33.8 & 37.5 & 38 & 37 & 165 & 13.4 & 13.5 & 13.2 \\
\hline $\mathrm{U}$ & 7.6 & 2.7 & 21.8 & 8.3 & 15.8 & 32.7 & 16.7 & 4.6 & 4.3 & 4.3 \\
\hline$\sum$ REE & 301.8 & 155.8 & 150.5 & 175.8 & 329.4 & 87.87 & 792.3 & 201.7 & 224.9 & 231.1 \\
\hline LREE & 282.69 & 138.37 & 137.87 & 103.54 & 272.77 & 73.42 & 747.8 & 169.68 & 198.26 & 201.77 \\
\hline HREE & 19.09 & 17.45 & 12.63 & 72.23 & 56,62 & 14,45 & 44,51 & 32.04 & 26.6 & 29.32 \\
\hline $\begin{array}{l}\text { LREE } \\
\text { /HREE }\end{array}$ & 14.8 & 7.9 & 10.9 & 1.4 & 4.8 & 5.1 & 16.8 & 5.3 & 7.5 & 6.9 \\
\hline $\mathrm{TE}_{1,3}$ & 1.04 & 0.96 & 1.03 & 1.18 & 1.14 & 1.15 & 1.04 & - & - & - \\
\hline $\mathrm{Eu} / \mathrm{Eu}^{*}$ & 0.6 & 0.57 & 0.44 & 0.003 & 0.07 & 0.1 & 0.05 & 0.62 & 0.67 & 0.62 \\
\hline
\end{tabular}

and - trachyandesite samples, grs - greisen samples, other explanations as in Table 2

The granites of the Tsagduul massif (TM) are similar in their major element proportions to the BGCh granites. They are characterized (based on the published data; op. cit.) by high contents of $\mathrm{SiO}_{2}$ (74.6-77.91\%). Somewhat variable values for alkali oxides are observed $\left(\mathrm{K}_{2} \mathrm{O} 2.64-5.41 \%, \quad \mathrm{Na}_{2} \mathrm{O}\right.$ $3.08-6.43 \%$ ), however the sum of these two values is similar in all the samples. The remaining major oxide contents are: $\mathrm{Al}_{2} \mathrm{O}_{3}$ 11.66-13.85\%, $\mathrm{Fe}_{2} \mathrm{O}_{3 \mathrm{t}}$ 0.4-1.19\%, $\mathrm{MgO} 0.02-0.2 \%, \mathrm{CaO}$ $0.11-0.84 \%, \mathrm{TiO}_{2}<0.01-0.16 \%, \mathrm{P}_{2} \mathrm{O}_{5} 0.03-0.07 \%$. In the case of the trachyandesites from the $\mathrm{BGCh}$ area, the following contents of the elements have been obtained: $\mathrm{SiO}_{2} 54.02-55.19 \%$, $\mathrm{Al}_{2} \mathrm{O}_{3}$ 16.22-17.71\%, $\mathrm{Fe}_{2} \mathrm{O}_{3 \mathrm{t}} 8.41-8.87 \%, \mathrm{MgO} 2.26-2.78 \%$, $\mathrm{MnO} 0.11-0.14 \%, \mathrm{CaO} 2.76-5.76 \%, \mathrm{Na}_{2} \mathrm{O} 3.21-4.31 \%, \mathrm{~K}_{2} \mathrm{O}$ 2.58-3.07\%, $\mathrm{TiO}_{2} 1.49-1.55 \%, \mathrm{P}_{2} \mathrm{O}_{5} 0.64-0.68 \%$ (Table 3).
Apart from the above-mentioned rocks, Table 3 comprises also the results of three geochemical analyses conducted for the greisens, common within the BGCh pluton. The samples analysed are a gray greisen with prevalent quartz, minor white mica and accessory chlorite (sample KW5); a light, quartz-white mica greisen (sample KW15) and a dark, biotitic greisen (sample MW31). The $\mathrm{SiO}_{2}$ content in the greisens is strongly scattered, from $55.77 \%$ in case of dark, botitic greisen, up to $89.66 \%$ for the light, quartzose type. Accordingly, the proportions of all the major elements in the greisens are also conspicuously variable.

The Harker diagrams plotted for the granitoids and trachyandesites from the areas compared display negative trends for $\mathrm{SiO}_{2}$ in relation to $\mathrm{Al}_{2} \mathrm{O}_{3}, \mathrm{MgO}, \mathrm{CaO}, \mathrm{FeO}_{\mathrm{t}}, \mathrm{TiO}_{2}$ and 

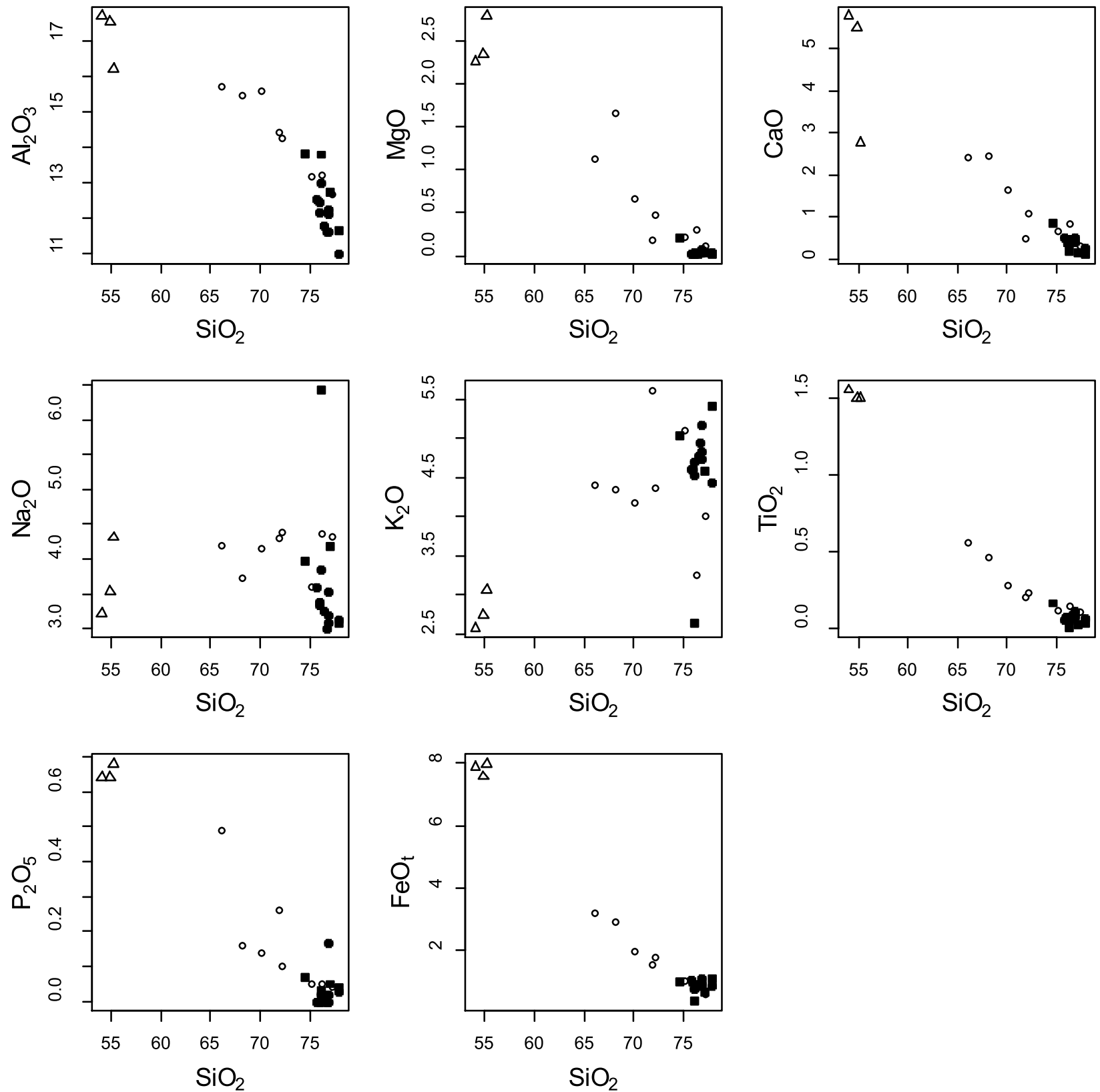

Fig. 3. Variation of $\mathrm{Al}_{2} \mathrm{O}, \mathrm{Na}_{2} \mathrm{O}, \mathrm{K}_{2} \mathrm{O}, \mathrm{CaO}, \mathrm{MgO}, \mathrm{TiO}_{2}, \mathrm{FeO}_{t} \mathrm{P}_{2} \mathrm{O}_{5}$ vs. $\mathrm{SiO}_{2}$ in the Baga-Gazriin Chuluu, Egiindavaa, Tsagduul granitoids and trachyandesites in the Baga-Gazriin Chuluu

Black circles - Baga-Gazriin Chuluu granites, open circles - Egiindavaa granitoids, black squares - Tsagduul granites, open triangles - trachyandesites

$\mathrm{P}_{2} \mathrm{O}_{5}$ (Fig. 3). In the case of the alkali elements, no clear trends are observed. Instead, the diagrams show considerable scattering of the projection points.

Concerning the trace elements, significant variability of their contents can be observed in the rocks from all the plutons compared. The Egiindavaa granitoids contain more $\mathrm{Ba}, \mathrm{Sr}$ and $\mathrm{Zr}$ than the Baga-Gazriin Chuluu and Tsagduul granites. In the EC rocks (based on the published data; op. cit), Ba ranges between 173-1430 ppm (av. 677 ppm), Sr 7.5-389 ppm (av.191 ppm), Zr 62-262 (av. 145 ppm); in the BGCh granites $\mathrm{Ba}$ is between 10-156 ppm (av. 55 ppm), Sr 7-24 ppm (av. 14 ppm), Zr 100-175 (av. 143 ppm). Finally, in the Tsagduul granites: $\mathrm{Ba}$ 0-246 ppm (av. $71 \mathrm{ppm}$ ), $\mathrm{Sr}$ 3.9-104 ppm (av. 30 ppm), Zr 61-142 ppm (av. 105 ppm). On the other hand, the EC granitoids are characterized by considerably lower values of $\mathrm{Rb}$ and $\mathrm{Y}$. In the EC granitoids, $\mathrm{Rb}$ equals 58-201 ppm (av. 135 ppm) and Y 10-23 ppm (av. 17 ppm) while in the BGCh granites Rb is 316-534 ppm (av. 419 ppm), $\mathrm{Y}$ 69-134 ppm (av.101 ppm) and in the TM granites Rb is 298-1150 ppm (av. 722 ppm), Y 19-108 ppm (av. 70 ppm). In the Harker (1900) diagrams plotted for the trace elements selected, no unequivocal trends are visible (Fig. 4). 

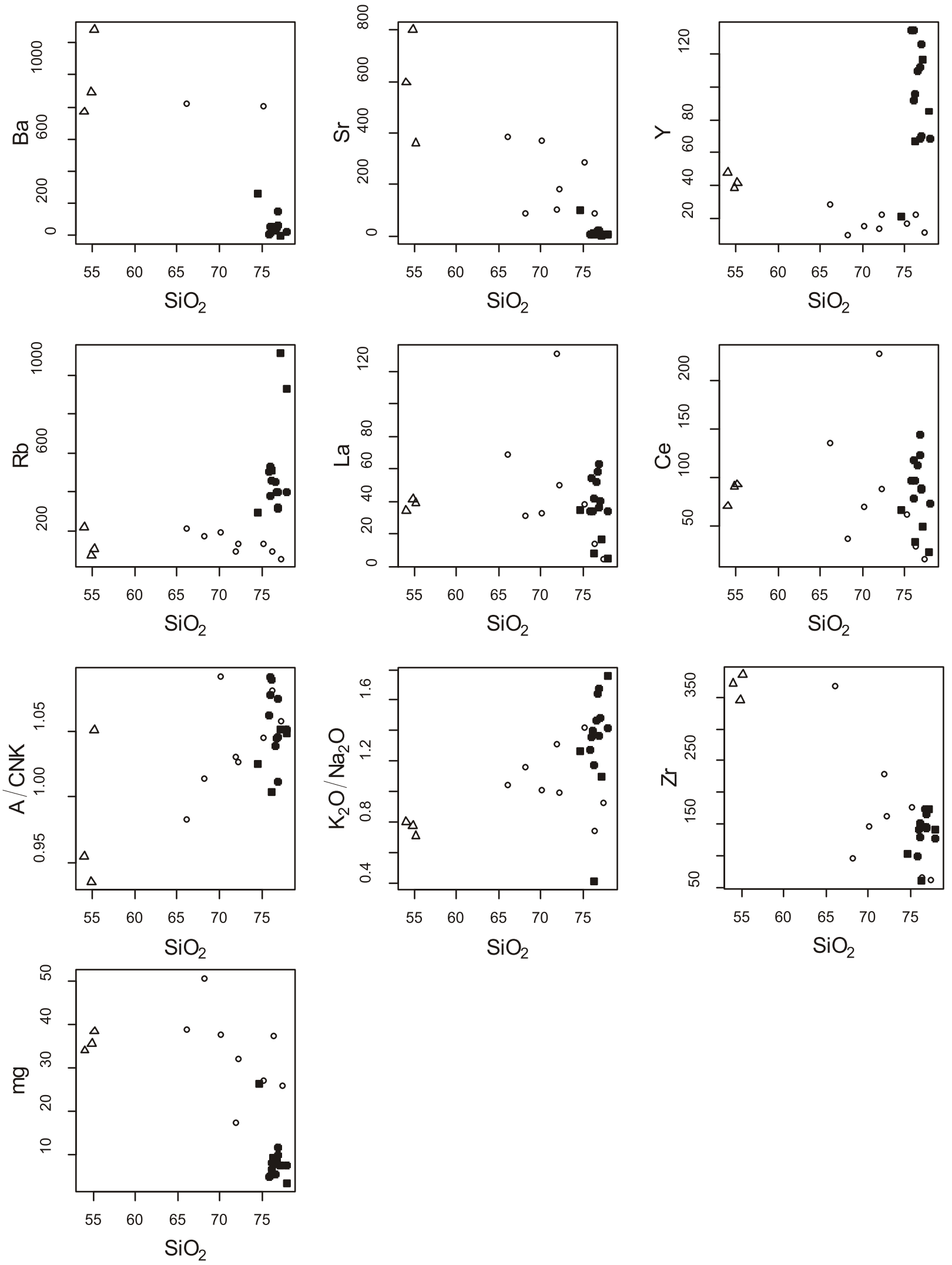

Fig. 4. Variation of $\mathrm{Ba}, \mathrm{Rb}, \mathrm{Sr}, \mathrm{La}, \mathrm{Y}, \mathrm{Ce}, \mathrm{Zr}, \mathrm{A} / \mathrm{CNK}\left(\operatorname{molar} \mathrm{Al}_{2} \mathrm{O}_{3} /\left(\mathrm{CaO}+\mathrm{Na}_{2} \mathrm{O}+\mathrm{K}_{2} \mathrm{O}\right), \mathrm{K}_{2} \mathrm{O} / \mathrm{Na}_{2} \mathrm{O}, \mathrm{mg}(\mathrm{Mg}\right.$ number$)$ vs. $\mathrm{SiO}_{2}$ for granitoids and trachyandesites 


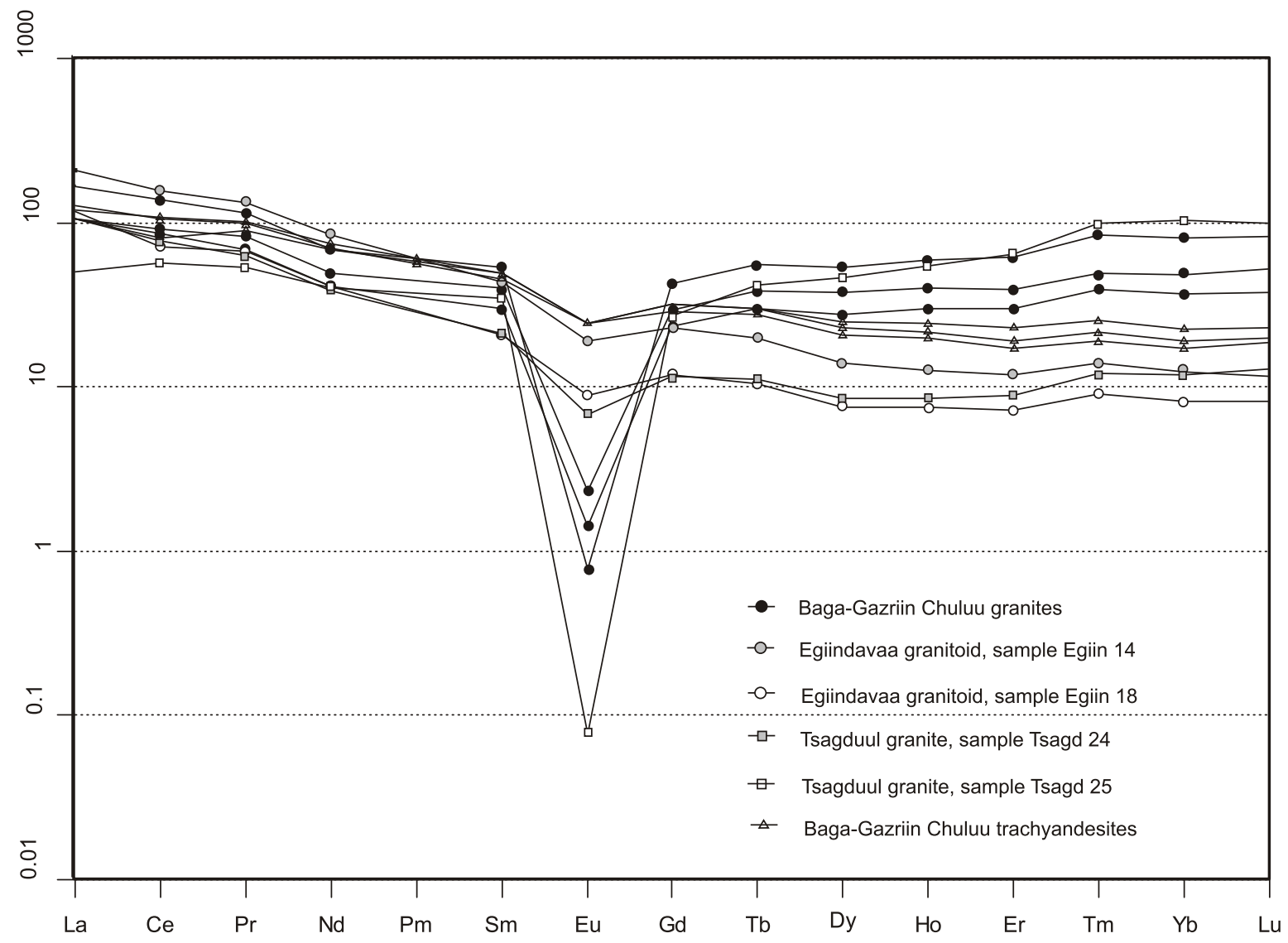

Fig. 5. Chondrite-normalized REE plots of granites and trachyandesites (normalized to values given in Nakamura, 1974)

\section{RARE-EARTH ELEMENTS AND SPIDER DIAGRAMS}

The BGCh granites display considerably increased values of rare earth element totals ( $\sum$ REE 185-323 ppm, av. 235 ppm), with LREE: 148-279 ppm (av. 209 ppm), HREE: 37-74 ppm (av. $53 \mathrm{ppm}$ ) and low LREE/HREE ratio: 3.1-6.4 (av. 4.1; Table 2). For the EC granitoids, the results of only two rare earth element analyses have been published (Oyungerel and Ishihara, 2005). The $\sum$ REE values of these two samples are respectively $156 \mathrm{ppm}$ and $302 \mathrm{ppm}$, including LREE: $138 \mathrm{ppm}$ and $283 \mathrm{ppm}$ and HREE $17.4 \mathrm{ppm}$ and $19.1 \mathrm{ppm}$, LREE/HREE ratio: 7.9 and 14.8 (Table 3 ). In case of the TM granites, the concentrations of the REE for only two samples also are given (Oyungerel and Ishihara, op cit.). Their total REE values are: 151 and $176 \mathrm{ppm}$ respectively, LREE equal: 104 and 138 ppm, whereas HREE: 13 and $72 \mathrm{ppm}$. The LREE/HREE ratios are 10.9 and $1.4 \mathrm{ppm}$ (Table 3). For the greisens, the $\sum$ REE contents range between 88 and 792 ppm (av. 403.5 ppm), with LREE: 74-748 ppm (av. 365 ppm), HREE: 14.5-57 ppm (av. 38.5 ppm) and LREE/HREE: 4.8-16.8 ppm (av. 8.9 ppm).

For the trachyandesites, the REE contents range between 202 and 231 ppm (av. 219 ppm), with LREE: 170-202 ppm (av. 190 ppm), HREE: 27-32 ppm (av. 29 ppm) and LREE/HREE: 5.3-7.5 ppm (av. 6.6 ppm).

In the rare earth element variation diagram (chondrite-normalized REE, Nakamura 1974; Fig. 5), the granitic rocks from the BGCh pluton are characterized by small enrichments in
LREE in relation to HREE, which display almost flat patterns, and by the conspicuous negative europium anomaly, typical for highly evolved A-type granites. A similar pattern is observed for one granite sample (Tsagd 25) from the Tsagduul massif. The second sample from this location (Tsagd 24) shows a weaker negative europium anomaly (referring to the BGCh granites and the Tsagd 25 sample), coupled with significant HREE depletion. Its projection in the REE variation diagram is more similar to the EC granitoids and BGCh trachyandesites (Fig. 5), than to the BGCh granites. The EC granitoids and the BGCh trachyandesites, show similar REE patterns (Fig. 5). They show fairly strong fractionation of REE in relation to HREE and display only a weak negative europium anomaly.

In the BGCh granites and in one granitic sample from the Tsagduul area (Tsagd 25), a distinct lanthanide tetrad effect (TE) has been observed (Fig. 5; e.g., Masuda and Ikeuchi, 1979; Masuda et al., 1987; Bau, 1996; Irber 1997, 1999; Zhenhua et al., 2002; Ishihara et al., 2008). The $\mathrm{TE}_{1,3}$ values for these rocks are higher than 1.1 (from 1.11 to 1.20 , Tables 2 and 3). Very weak tetrad effect, inconspicuous on the REE diagrams, is noted also in the second Tsagduul sample (Tsagd 24, $\left.\mathrm{TE}_{1,3}=1.03\right)$ and in one of the samples from the Egiindavaa complex (Egiin 14, TE $\left._{1,3}=1.04\right)$.

The rocks investigated have been also interpreted using spider variation diagrams (Figs. 6-7, normalized to primitive mantle; Sun and McDonough, 1989). In the diagram normalized to primitive mantle, the BGCh granites display distinct de- 


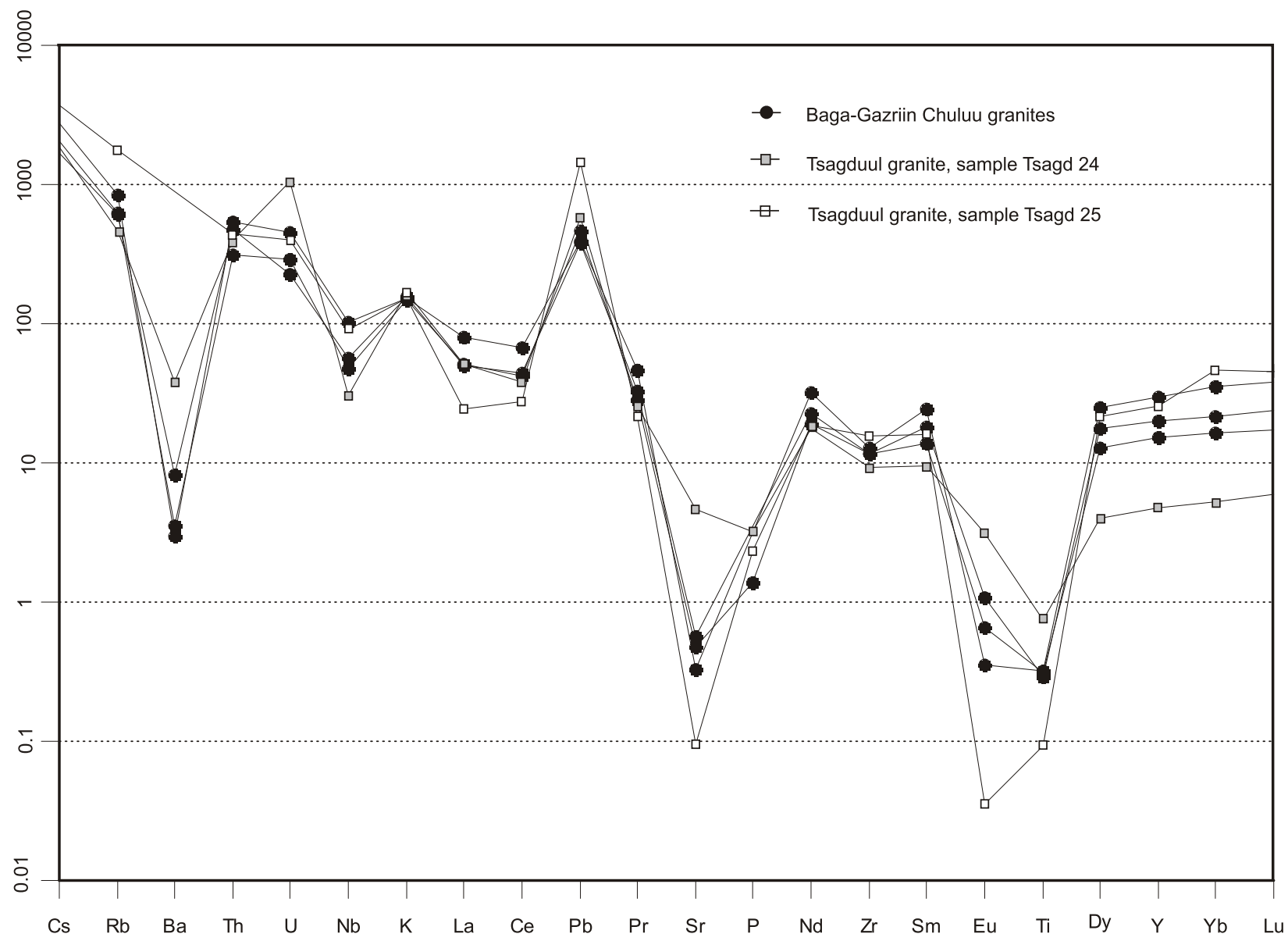

Fig. 6. Spider diagram of Baga-Gazriin Chuluu and Tsagduul granites normalized to primitive mantle (Sun and McDonough, 1989)

pletion in $\mathrm{Ba}, \mathrm{Sr}$ and $\mathrm{Ti}$ and slight enrichment in Csand $\mathrm{Pb}$ (Fig. 6). Similar signatures can be observed for one of the samples from the Tsagduul area (Tsagd 25). Its pattern is characterized by more distinct depletion in $\mathrm{Sr}, \mathrm{Eu}$ and $\mathrm{Ti}$ (very strong negative anomalies) and lack of measurable $\mathrm{Ba}$. The Tsagd 25 enrichment in $\mathrm{Cs}, \mathrm{Rb}, \mathrm{Pb}$ and $\mathrm{Yb}$ is also higher than that in the BGCh granites. The second sample of the Tsagduul granite (Tsagd 24) shows a different pattern. The negative anomalies of $\mathrm{Ba}, \mathrm{Sr}$ and $\mathrm{Ti}$ are much weaker than in the case of the remaining samples shown in the diagram. Sample Tsagd 24 (compared to the BGCh granites) also shows small depletion in $\mathrm{Nb}$ and $\mathrm{Zr}$, significant depletion in $\mathrm{Dy}, \mathrm{Y}, \mathrm{Yb}$ and $\mathrm{Lu}$ and weak enrichment in U. The two samples shown of the EC granitoids (Fig. 7) reveal more or less similar overall patterns, however, they differ in concentrations of particular elements. The first sample (Egiin 14) in comparison to the second one (Egiin 18) displays increased values of almost all elements, with the most distinct enrichment in $\mathrm{Cs}$, Th, $\mathrm{U}$ and in the elements from $\mathrm{Sr}$ to $\mathrm{Lu}$. It is also characterized by weak negative $\mathrm{Ba}, \mathrm{Nb}$ and $\mathrm{Sr}$ anomalies and conspicuous depletion in Ti. Sample Egiin 18 (in relation to Egiin 14) is significantly depleted in all elements, especially in $\mathrm{Nb}, \mathrm{P}, \mathrm{Ti}$. The depletion, visible in the right part of the diagram, makes the Egiindavaa granitoids similar to sample Tsagd 24 (Fig. 6). The BGCh trachyandesites give similar patterns for all three samples analysed (Fig. 7); however, one of the samples contains slightly increased concentrations of Cs and $\mathrm{Rb}$. All these trachyandesites are characterized by a negative $\mathrm{Nb}$ anomaly and inconspicuous depletion in Ti. Compared with the Egiindavaa granitoids, they possess higher contents of $\mathrm{Dy}, \mathrm{Y}, \mathrm{Yb}$ and $\mathrm{Lu}$.

\section{A-TYPE GRANITES}

Discrimination of the A-type granites is problematic and complex. Indeed, there is still no clear criterion for distinguishing this group of rocks. Some petrologists distinguish A-type granites using tectonic context. They interpret them as within-plate (e.g., Pearce et al., 1984), anorogenic (Maniar and Piccoli, 1989) or post-collisional rocks (Sylvester, 1989). Frequently they are additionally divided into subtypes (e.g., Eby, 1992). The other discrimination criterion, sometimes complementary to the tectonic setting of the granite, is its geochemical profile. The A-type granites are characterized as anhydrous, alkaline, but sometimes also aluminous rocks. The problem of classification of these specific granites has been discussed in detail in many publications (e.g., Collins et al., 1992; Klimm et al., 2003; Vander Auwera et al., 2003; Frost and Frost, 2011).

The granitoid rocks compared here, using our data for the Baga-Gazriin Chuluu (BGCh) pluton and the published geochemical data for the Egiindava Complex (EC) and Tsagduul massif (TM; Oyungerel and Ishihara, 2005), have been inter- 


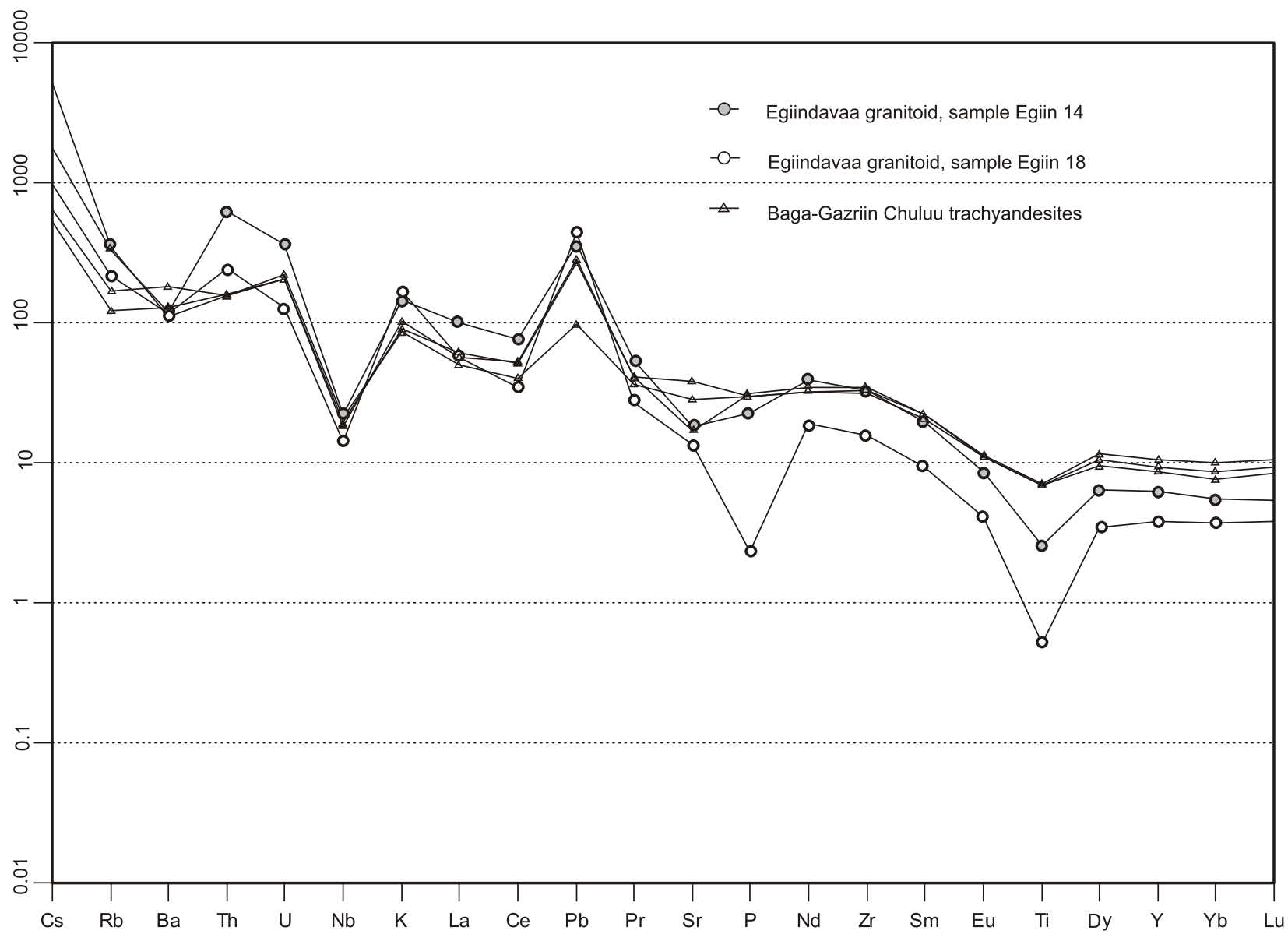

Fig. 7. Spider diagram of Egiindavaa granitoids and Baga-Gazriin Chuluu trachyandesites normalized to primitive mantle (Sun and McDonough, 1989)

preted on several classification diagrams. Using the plots of Whalen et al. (1987), the BGCh and TM granites in all cases occupy the fields of the A-type granites (Fig. 8). On the other hand, the EC granitoids are mostly located within the I- and Stype granite field.

Referring to the classification of A-granites proposed by Frost et al. (2001), Frost and Frost (2011) and graphically represented by the $\mathrm{FeO}_{\mathrm{t}}\left(\mathrm{FeO}_{\mathrm{t}}+\mathrm{MgO}\right) v s . \mathrm{SiO}_{2}$ diagram (Fig. 9A), the BGCh granites and TM granites plot in the field of ferroan granites, whereas the EC granitoids are located both in the fields of ferroan (3 samples) and magnesian granites (5 samples). In the MALI diagram (modified alkali lime index; $\mathrm{Na}_{2} \mathrm{O}$ $+\mathrm{K}_{2} \mathrm{O}-\mathrm{CaO}$ vs. $\mathrm{SiO}_{2}$ ) after Frost et al. (2001), Frost and Frost (2011), the granitoids studied occupy the position of alkalic and calc-alkalic granites (Fig. 9B), but the alkalic field is occupied solely by the samples from Egiindavaa. As shown by experimental research (Holtz and Johannes, 1991; Patiňo Douce and Harris, 1998), the MALI index increases simultaneously with decrease of water content. In the case of the EC granitoids, it appears, that the MALI index increases together with the differentiation of melt, indicating a decreasing water content over time.

Based on the data presented, the BGCh granites and the majority of the TM granites can be classified as the A-type granites, which typically occur in the anorogenic, intracontinental settings and are usually enriched in rare earth elements (Frost and Frost, 2011). Only a minority of the EC granitoids belong to the A-type granites (three out of eight samples analysed).

\section{DISCUSSION}

The Central Mongolian granitoids compared from the three plutonic massifs of similar age and situated relatively close to each other, do not display identical geochemical profiles.

The BGCh granites are regarded as having been generated at shallow levels in continental crust, as shown by their geochemical profile. Simultaneously, the temperature range obtained $\left(\mathrm{ca} .800^{\circ} \mathrm{C}\right.$, zircon thermometer by Watson and Harrison (1983; Tables 2 and 3) of the BGCh magma formation seems to be high for such small depths. They were the product of crystallisation of completely melted magma (no enclaves) having (in the initial stages) low water contents. The small amount of water is shown also by the relatively weakly developed contact envelope. The interstitial position of micas between feldspars and quartz may testify to their late crystallisation. The presence of REE minerals in the dark micas together with exceptionally small size of the zircons (concentrates of grains $>50$ micrometers for SHRIMP dating could not be obtained) may indicate enrichment of the melt in complex ions, which were the carriers of the REE in the late stage of crystallisation. This enrichment resulted also in the occurrence of elements with po- 

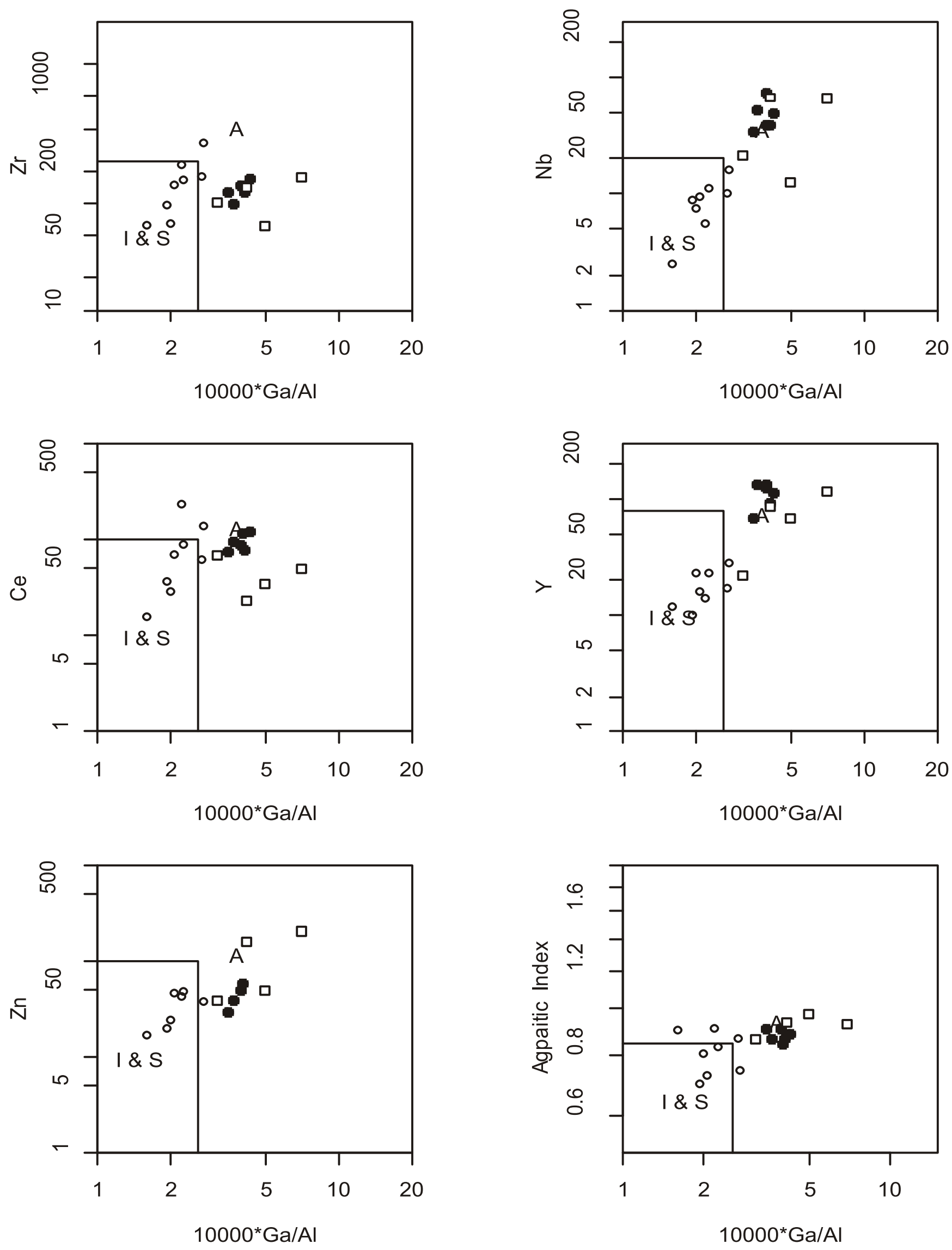

Fig. 8. Geotectonic diagrams of A-type granitoids (Whalen et al., 1987)

Black circles - Baga-Gazriin Chuluu granites, open circles - Egiindavaa granitoids, open squares - Tsagduul granites 

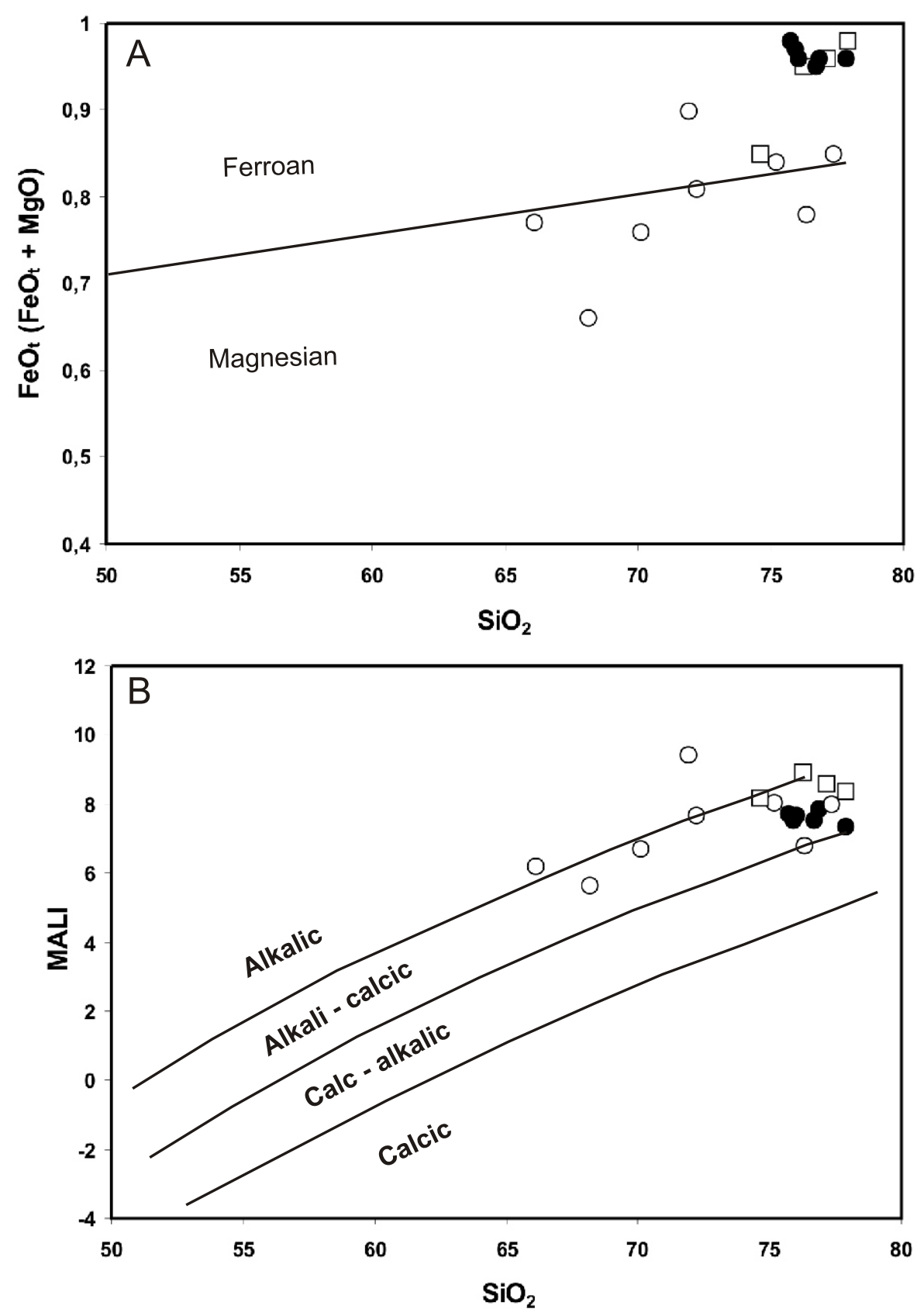

Fig. 9A - $\mathrm{FeO}_{\mathrm{t}} / \mathrm{FeO}_{\mathrm{t}}+\mathrm{MgO}_{\mathrm{t}}$ vs. $\mathrm{SiO}_{2}$ diagram (Frost et al., 2001), B - MALI (modified alkali-lime index: $\mathrm{Na}_{2} \mathrm{O}+\mathrm{K}_{2} \mathrm{O}-\mathrm{CaO}$ ) vs. $\mathrm{SiO}_{2}$ diagram (Frost et al., 2001)

Explanations as in Figure 3

tential economic significance (e.g., W and Sn). All the varieties of the BGCh granites reveal high levels of the "tetrad effect" $\left(\mathrm{TE}_{1,3}\right)$. It is hard to constrain whether the inferred significant amount of the fluids delivered was connected exclusively with the host magma and was generated due to melting of the upper crust (Bettencourt et al., 2005), or whether it was activated as an effect of degassing of the uppermost part of the metasomatised mantle (Charoy and Raimbault, 1994). Undoubtedly, the fluids migrated through channel systems connected with fractures (e.g., Azzouni-Sekkal et al., 2003). Field observations suggest that the distribution of the greisens in the BGCh area is determined by the presence of joint systems.

The BGCh granites display geochemical affinity to the majority of the TM granites. The data on the major and chosen trace elements of the TM granites based on Ishihara and Oyungerel (2005), are shown in the diagrams (Figs. 3-9) and in the Tables 2 and 3. Almost all the TM rocks, except for sample Tsagd 24, belong to the A-type granites and could be generated by a mechanism analogical to that of the BGCh pluton. Also, the possibility of their formation in the upper part of the conti- 
nental crust, without- or with only minor amounts of mantle material, seems to be plausible.

The EC granitoids reveal features typical of hybrid magmas, thus only in part they can be treated as A-type granites. The distinct internal diversity of the EC granitoids can be observed already at the level of macroscopic observations. The Egiindavaa pluton includes both leucogranites and hornblende-bearing granitoids (Oyungerel and Ischihara, 2005), which explains the large inhomogeneity in its geochemical characteristics. The EC rocks show possible transition from granitoids with mantle magma signatures (higher concentrations of $\mathrm{Fe}_{2} \mathrm{O}_{3}, \mathrm{CaO}, \mathrm{P}_{2} \mathrm{O}_{5}, \mathrm{Sr}, \mathrm{Eu}$ ) to typical, highly evolved A-type granites, with much less discernible mantle influence.

However, the internal diversity of the EC granitoids may be also the result of the erroneous classification of two genetically separate intrusions (hybridal granite and A-type granite) formed in the different magmatic episodes, to one plutonic body (the Egiindavaa complex). On the other hand, in the light of the comparison made here, the distinguishing of the TM granites into a separate group reflects rather geographic distance, than genetic differences between the A-type granites included into the Edingavaa complex and Tsagduul massif. The possible mistakes in qualifying the rocks to particular regional units (EC and TM) may result, as Oyungerel and Ishihara (2005) admit, from ambiguous field relationships due to the poor exposure of the granites studied.

The similarity of the trends in the REE and spider diagrams (Figs. 5-7) between the most of the EC granitoids and the trachyandesites from the BGCh neighbourhood may support the presence of mantle-derived material in the EC melt. A significant water content during the initial stages of magma crystallisation for the majority of the EC granitoids is shown both by their mineral composition (large amounts of amphiboles and micas) and MALI index values (Fig. 9B).

\section{CONCLUSIONS}

Comparison of geochemical data shows that granites of the Baga-Gazriin Chuluu pluton and the A-type granites of the Egiindavaa complex and Tsagduul massif (from the Hotont area) could have formed at the same time.

On the other hand, the majority of the rocks defined by Oyungerel and Ischihara (2005) as the EC granitoids has been generated in distinctly different conditions. They formed with the unquestionable contribution of mantle material in the melt, perhaps earlier than the remaining A-type granites of this area. Hypothetically, without precise radiometric dating, they may be even attributable to a subduction regime.

Based on the results of geological mapping, the trachyandesites neighbouring the BGCh granitic pluton seem to belong to the Permian volcano-sedimentary complex, showing concordant boundaries with the strata of that Late Paleozoic succession.
The hydrothermal alternation in the trachyandesites adjacent to the BGCh pluton seems to confirm that their position has not changed since the intrusion of the granites.

Firm conclusions cannot be drawn without radiometric dating of the plutons described, because the field relationships between them do not allow for unequivocal inerpretation.

All the A-type granites discussed in this paper were generated in decompressional conditions, connected with the extension of continental crust, which followed the closure of the westernmost part of the Mongol-Okhotsk Ocean. A shallow-lying source of intense heat, necessary for the onset of magmatic processes in the upper level of the continental crust, may have been connected with melting of the subducted slab after its break-off and the subsequent ascent of the magma generated. A similar model has been proposed for other regions in central Asia (Liu, 2005; Zorin et al., 2006).

An alternative model could invoke the presence of mantle plume under the area investigated. Regardless of the cause, the existence of a shallow-lying heat source in this region in the Early Mesozoic seems to be beyond doubt. Although most of the recent publications on the Phanerozoic granitoids of central Asia, which use neodymium isotope analyses, indicate the "juvenile" character and strong mantle signatures of these rocks (e.g., Jahn et al., 2000, Kovalenko et al., 2004), based on the results of the interpretation of geochemical analyses of the Baga-Gazriin Chuluu area and the Hotont area granites (without isotopic data), there seems little support for the direct contribution of the mantle-derived material in the magmas which formed the A-type granites studied. Perhaps the role of mantle magmas was limited to provide the heat source which warmed from beneath the upper levels of the crust, resulting in its melting.

It may be inferred that the sequence of events, from the functioning of the Mongol-Okhotsk Ocean (at least in the early Carboniferous), through its final closure (eastern part in the Jurassic, western part much earlier - ?P/T), formation of a tectonic suture and extension of the crust coupled with the generation of A-type granites (T/J) seems to be plausible and is consistent with the ascent of mantle-derived magmas into the shallower parts of the continental crust.

Acknowledgements. The reasearch was funded by Polish Ministry of Science and High Education grant no. N N307 225835. M. K. benefitted from a financial support of the DAAD (German academic exchange office; agreement between Poznań and Hannover Universities). The authors address sincere thanks to Dr. D. Batulzii of the School of Geology and Petroleum Engineering at the Mongolian University of Science and Technology, for provision of helpful cartographic materials concerning the study area and for valuable advice during the organization of fieldwork. They thank also Mr. S. Boldbaatar, the General Director of the Jules Vernes Mongolia Co., for his great help in the organization of the expeditions, and for logistic facilities, transfer of the samples to Poland and great kindness. The authors are also very grateful to L. Krzemiński and $\mathrm{V}$. Hoeck for their revision and constructive comments that helped to improve this paper. 


\section{REFERENCES}

AZZOUNI-SEKKAL A., LIÉGOIS J.P., BECHIRI-BENMERZOUG F., BELAIDI-ZINET S. and BONIN B. (2003) - The "Taourirt" magmatic province, a marker of the closing stages of Pan-African orogeny in the Taureg Shield: review of the available data and Sr-Nd isotope evidence. J. Afr. Earth Sc., 37: 337-350.

BADARCH G. and TOMURTOGOO O. (2001) - Tectonostratigraphic Terranes of Mongolia. Gondwana Res., 4 (2): 143-144.

BADARCH G., CUNNINGHAM W.D. and WINDLEY B.F. (2002) - A new terrane subdivision for Mongolia: implications for the Phanerozoic crustal growth of Central Asia. J. Asian Earth Sc., 21: $87-110$.

BATULZII D., GANTUMUR H. and GANDUSH B. (2003) - Late Mesozoic volcanic activity in Mongolia: Implications for post-collisional magmatic evidence resulting from the closure of Mongol-Okhotsk oceanic basin. In: Proc. of the JAGOD Conference, Vladivostok: $77-79$.

BAU M. (1996) - Controls on the fractionation of isovalent trace elements in magmatic and aqueous systems: evidence from $\mathrm{Y} / \mathrm{Ho}, \mathrm{Zr} / \mathrm{Hf}$, and lanthanide tetrad effect. Contrib. Miner. Petrol., 123: 323-333.

BETTENCOURT J.S., LEITE W.B. jr., GORAIEB C.L., SPARRENBERGER I., BELLO R.M.S. and PAYOLLA B.L. (2005) Sn-polymetallic greisen-type deposits associated with late-stage rapakivi granites, Brazil: fluid inclusion and stable isotope characteristics. Lithos, 80: 363-386.

BUCHAN C., PFÄNDER J., KRÖNER A., BREWER T.S., TOMURTOGOO O., TOMURHUU D., CUNNINGHAM D. and WINDLEY B.F. (2002) - Timing of accretion and collisional deformation in the Central Asian Orogenic Belt: implications of granite geochronology in the Bayankhongor Ophiolite Zone. Chem. Geol., 192 23-45.

BUSLOV M.M., SAPHONOVA I.YU, WATANABE T., OBUT O.T., FUJIWARA Y., IWATA,K., SEMAKOV N.N., SUGAI Y., SMIRNOVA L.V., KAZANSKY A.YU. and ITAYA T. (2001) - Evolution of the Paleo-Asian Ocean (Altai-Sayan Region, Central Asia) and collision of possible Gondwana-derived terranes with the southern marginal part of the Siberian continent. Geosc. J., 5: 203-224.

CAWOOD P.A., KRÖNER A., COLLINS W.J., KUSKY T.M., MOONEY W.D. and WINDLEY B.F. (2009) - Accretionary orogens through Earth history. Geol. Soc., Spec. Publ., 318: 1-36.

CHAROY B. and RAIMBAULT L. (1994) - Zr-, Th-, and REE-rich biotite differentiates in the A-type granite pluton of Suzhou (Eastern China): the key role of fluorine. J. Petrol., 35 (4): 919-962.

COGNE J.P., KRAVCHINSKY V. A., HALIM N. and HANKARD F (2005) - Late Jurassic-Early Cretaceous closure of the Mongol-Okhotsk Ocean demonstrated by new Mesozoic palaeomagnetic results from the Trans-Baikal area (SE Siberia). Geoph. J. Internat., 163: 813-832.

COLLINS W.J., BEAMS S.D., WHITE A.J.R. and CHAPPELL B.W. (1992) - Nature and origin of A-type granites with particular reference to southeastern Australia. Contrib. Miner. Petrol., 80: 189-200.

De la ROCHE H., LETERRIER J., GRANDCLAUDE P. and MARCHAL M. (1980) - A classification of volcanic and plutonic rocks using R1R2-diagram and major element analyses - its relationships with current nomenclature. Chem. Geol., 29: 183-210.

EBY G.N. (1992) - Chemical subdivision of the A-type granitoids: petrogenetic and tectonic implications. Geology, 20: 641-644.

FROST B.R., BARNES C.G., COLLINS W.J., ARCULUS R.J., ELLIS D.J. and FROST C.D. (2001) - A geochemical classification for granitic rocks. J. Petrol., 42 (11): 2033-2048.

FROST C.D. and FROST B.R. (2011) - On ferroan (A-type) granitoids: their compositional variability and models of origin. J. Petrol., 52: 39-53.

HARKER A. (1900) - Igneous rock series and mixed igneous rocks. J. Geol., 8: 359-399.

HOLTZ F. and JOHANNES W. (1991) - Genesis of peraluminous granites II. Mineralogy and chemistry of the Tourem complex (North Portugal). Sequential melting $v s$. restite unmixing. J. Petrol., 32: 959-978.
IRBER W., FÖRSTER H.J., HECHT L., MÖLLER P. and MORTEANI G. (1997) - Experimental, geochemical, mineralogical and O-isotope constraints on the late-magmatic history of the Fichtelgebirge granites (Germany). Geol. Rundsch., 86 (suppl.): 110-124.

IRBER W. (1999) - The lanthanide tetrad effect and its correlation with $\mathrm{K} / \mathrm{Rb}, \mathrm{Eu} / \mathrm{Eu}^{*}, \mathrm{Sr} / \mathrm{Eu}, \mathrm{Y} / \mathrm{Ho}$, and $\mathrm{Zr} / \mathrm{Hf}$ of evolving peraluminous granite suites. Geochim. Cosmochim. Acta., 63 (3/4): 489-508.

ISHIHARA S., RENMIN H., HOSHINO M. and MURAKAMI H. (2008)REE abundance and REE minerals in granitic rocks in the Nangling Range, Jingxi province, Southern China, and generation of the REE-rich weathered crust deposits. Resour. Geol., 58 (4): 355-372.

JAHN B.M. (2004) - The Central Asian Orogenic Belt and growth of the continental crust in the Phanerozoic. Geol. Soc. London, Spec. Publ., 226: $73-100$.

JAHN B., WU F. and CHEN B. (2000) - Massive granitoid generation in Central Asia: $\mathrm{Nd}$ isotope evidence and implication for continental growth in the Phanerozoic. Episodes, 23 (2): 82-92.

JANOUŠEK V., FARROW C.M. and ERBAN V. (2006) - Interpretation of whole-rock geochemical data in igneous geochemistry: introducing Geochemical Data Toolkit (GCDkit). J. Petrol., 47 (6): 1155-1259.

KELTY T.K., YIN A., BATULZII D., GEHRELS G.E. and RIBEIRO A.E. (2008) - Detrital-zircon geochronology of Paleozoic sedimentary rocks in the Hangay-Hentey basin, north-central Mongolia: implications for the tectonic evolution of the Mongol-Okhotsk Ocean in central Asia. Tectonophysics, 451: 290-311.

KLIMM K., HOLTZ F., JOHANNES W. and KING P. L. (2003) - Fractionation of metaluminous A-type granites: an experimental study of the Wangrah Suite, Lachlan Fold Belt, Australia. Precambrian Res., 124: 327-341.

KOVALENKO V.I., KUZMIN M.I., ZONENSHAIN L.P., NAGIBNA M.S., PAVLENKO A.S., VLADIKIN N.B., TSEDEN TS., GUNDSAMBY TS. and GOREGLYAD A.V. (1971) Baga-Gazrinskij masiv granitov shakharadinskovo tipa i odnoimiennoye olovianoye mestorozhdeniye. Rare metal granitoids of Mongolia, Transaction, vol. 5 of Joint Soviet-Mongolian scientific research geological expedition, Nauka, Moscow, 61-69.

KOVALENKO V.I., YARMOLYUK V.V., KOVACH V.P., KOTOV A.B., KOZAKOV I.K., SALNIKOVA E.B. and LARIN A.M. (2004) - Isotope provinces, mechanism of generation and sources of the continental crust in the Central Asian mobile belt: geological and isotopic evidence. J. Asian Earth Sc., 23: 605-627.

LIU H. (2005) - Petrology, geochemistry and geochronology of late Triassic volcanics, Kunlun orogenic belt, western China: implications for tectonic setting and petrogenesis. Geochem. J., 39: 1-20.

MANIAR P.D. and PICCOLI P.M. (1989) - Tectonic discrimination of granitoids. Geol. Soc. Am. Bull., 101: 635-643.

MASUDA A. and IKEUCHI Y. (1979) - Lanthanide tetrad effect observed in marine environments. Geochem. J., 13: 19-22.

MASUDA A., KAWAKAMI O., DOHMOTO Y. and TAKENAKA T. (1987) - Lanthanide tetrad effects in nature: two mutually opposite types W and M. Geochem. J., 21: 119-124.

MOSSAKOVSKY A.A., RUZHENTSEV S.V., SAMYGIN S.G. and KHERASKOVA T.N. (1994) - Central Asian Fold Belt: geodynamic evolution and formational history. Geotectonics, 27 (6): 445-474.

NAKAMURA N. (1974) - Determination of REE, Ba, Fe, Mg, Na and K in carbonaceous and ordinary chondrites. Geochim. Cosmochim. Acta, 38: $757-773$.

OYUNGEREL S. and ISHIHARA S. (2005) - Paleozoic and Mesozoic granitic rocks in the Hotong area, central Mongolia. Bull. Geol. Surv. Japan, 56 (7/8): 245-258.

PATIŇO DOUCE A.E. and HARRIS N. (1998) - Experimental constraints on Himalayan anatexis. J. Petrol., 39: 689-710.

PEARCE J.A., HARRIS N.B.W. and TINDLE A.G. (1984) - Trace element discrimination for the tectonic interpretation of granitic rocks. J. Petrol., 25: 956-983.

SUN S.S. and McDONOUGH W.F. (1989) - Chemical and isotopic systematics of oceanic basalts: implications for mantle composition and processes. Geol. Soc. Spec. Publ., London, 42: 313-345. 
SYLVESTER P.J. (1989) - Post-collisional alkaline granites. J. Geol., 97: 261-280.

TOMURTOGOO O. (1997) - A new tectonic scheme of the Paleozoides in Mongolia. Proc. 30th Internat. Geol. Congress, 7: 75-82.

TOMURTOGOO O., WINDLEY B.F., KRONER A., BADARCH G. and LIU D.Y. (2005) - Zircon age and occurrence of the Adaatsag ophiolite and Muron shear zone, central Mongolia: constraints on the evolution of the Mongol-Okhotsk ocean, suture and orogen. Geol. Soc. Spec. Publ., London, 162: 125-134.

VANDER AUWERA J., MARTIN H. and RÄMÖ T. (2003) - Origin and evolution of Precambrian anorogenic magmatism. Spec. Issue. Precambrian Res., 124 (2-4).

WATSON E.B. and HARRISON M., (1983) - Zircon saturation revisited: temperature and composition effects in a variety of crustal magma types. Earth Planet Sc. Lett., 64: 295-304.

WHALEN J.B, CURRIE K.L. and CHAPPELL B.W. (1987) - A-type granites: geochemical characteristics, discrimination and petrogenesis. Contrib. Miner. Petrol., 95: 407-419.
WINDLEY B.F., ALEXEIEV D., XIAO W., KRÖNER A. and BADARCH G. (2007) - Tectonic models for accretion of the Central Asian Orogenic belt. J. Geol Soc, London, 164: 31-47.

YAKUBCHUK A. (2008) - Re-deciphering the tectonic jigsaw puzzle of northern Eurasia. J. Asian Earth Sc., 32: 82-101.

XIAO W.J., HUANG B.C., HAN C.M., SUN S. and LI J.L. (2010) - A review of the western part of the Altaids: a key to understanding the architecture of accretionary orogens. Gondwana Res., 18: 253-273.

ZHENHUA Z., XIAOLIN X., XIAODONG H., YIXIAN W., QIANG W., ZHIWEI B. and JAHN B. (2002) - Controls on the REE tetrad effect in granites: Evidence from the Qianlishan and Baerzhe Granites, China. Geochem. J., 36: 527-543.

ZONENSHAIN L.P., KUZMIN M.I. and NATAPOV L.M. (1990) - Geology of the USSR: a plate tectonic synthesis. Am. Geoph. Union Geodynam. Ser., 21: 1-242.

ZORIN A.Y., TURUTANOV E.K., KOZHEVNIKOV V.M., RASSKAZOV S.V. and IVANOV A.V. (2006) - Cenozoic upper mantle plumes in east Siberia and central Mongolia and subduction of the Pacific plate. Dokl. Earth Sc., 409 (5): 723-726. 